\title{
OPTICAL SPECTROSCOPY OF X-RAY SOURCES IN THE EXTENDED CHANDRA DEEP FIELD SOUTH ${ }^{*, \dagger}$
}

\author{
Ezequiel Treister ${ }^{1,2,9}$, Shanil Virani $^{3}$, Eric Gawiser $^{4}$, C. Megan Urry $^{3,5}$, Paulina Lira $^{6}$, Harold Francke ${ }^{6}$, Guillermo $^{3}$ \\ A. Blanc ${ }^{7}$, Carolin N. Cardamone $^{3}$, MaAike Damen $^{8}$, Edward N. TaYlor ${ }^{8}$, And Kevin Schawinski ${ }^{3}$ \\ ${ }^{1}$ 1Institute for Astronomy, 2680 Woodlawn Drive, University of Hawaii, Honolulu, HI 96822, USA; treister@ifa.hawaii.edu \\ 2 2European Southern Observatory, Casilla 19001, Santiago 19, Chile \\ 3 3Department of Astronomy, Yale University, P.O. Box 208101, New Haven, CT 06520, USA \\ 4 4Department of Physics and Astronomy, Rutgers, 136 Frelinghuysen Road, Piscataway, NJ 08854-8019, USA \\ 5 5Department of Physics, Yale University, P.O. Box 208121, New Haven, CT 06520, USA \\ ${ }^{6}$ 6Departamento de Astronomía, Universidad de Chile, Casilla 36-D, Santiago, Chile \\ 7 7Department of Astronomy, University of Texas at Austin, 1 University Station, C1400 Austin, TX 78712, USA \\ 8 8Sterrewacht Leiden, Leiden University, NL-2300 RA Leiden, The Netherlands \\ Received 2008 July 7; accepted 2008 December 10; published 2009 March 10
}

\begin{abstract}
We present the first results of our optical spectroscopy program aimed to provide redshifts and identifications for the X-ray sources in the Extended Chandra Deep Field South. A total of 339 sources were targeted using the IMACS spectrograph at the Magellan telescopes and the VIMOS spectrograph at the VLT. We measured redshifts for 186 X-ray sources, including archival data and a literature search. We find that the active galactic nucleus (AGN) host galaxies have on average redder rest-frame optical colors than nonactive galaxies, and that they live mostly in the "green valley." The dependence of the fraction of AGNs that are obscured on both luminosity and redshift is confirmed at high significance and the observed AGN spatial density is compared with the expectations from existing luminosity functions. These AGNs show a significant difference in the mid-IR to X-ray flux ratio for obscured and unobscured AGNs, which can be explained by the effects of dust self-absorption on the former. This difference is larger for lower luminosity sources, which is consistent with the dust opening angle depending on AGN luminosity.
\end{abstract}

Key words: galaxies: active - quasars: general - X-rays: galaxies

Online-only material: color figure, machine-readable table

\section{INTRODUCTION}

X-ray surveys have been critical in unveiling a large population of obscured supermassive black holes actively accreting matter (e.g., Giacconi et al. 2001 and references therein), contrary to optical surveys, like the Sloan Digital Sky Survey (SDSS; e.g., Schneider et al. 2002), which are dominated by unobscured active galactic nuclei (AGNs). The hard spectral shape of the X-ray background (XRB; e.g., Gruber 1992) reveals that the obscured AGNs should significantly outnumber the unobscured sources, as was first proposed by Setti \& Woltjer (1989). Given the difficulties in detecting and identifying the low luminosity and/or high redshift AGNs, in particular those heavily obscured, it has been very challenging to obtain a complete census of the AGN population. This is particularly important in order to study the growth of supermassive black holes and its relation to the formation and evolution of its host galaxy.

Deep X-ray surveys like the Chandra Deep Fields North (CDF-N; Brandt et al. 2001) and South (CDF-S; Giacconi et al. 2001; Rosati et al. 2002) have been critical in obtaining a significant sample of obscured AGNs up to significant distances. The first results from the optical follow-up of the X-ray sources in the CDFs reported a peak of the AGN activity at $z \sim 0.7$ (Szokoly et al. 2004), in contradiction with the predictions of the early XRB population synthesis (e.g., Comastri et al. 1995; Gilli et al. 2001), which expected a redshift peak at $z \sim 1.5$. This discrepancy was resolved in the XRB population synthesis

\footnotetext{
* This paper includes data gathered with the $6.5 \mathrm{~m}$ Magellan Telescopes located at Las Campanas Observatory, Chile.

$\dagger$ Partly based on observations collected at the European Southern Observatory, Chile, under programs 078.A-0485 and 072.A-0139.

9 Chandra Fellow.
}

models of Treister \& Urry (2005) and later by Gilli et al. (2007), both of whom incorporated the latest Chandra and XMMNewton observational results in their calculations. Similarly, the observed fraction of obscured to unobscured AGNs in the CDFs was $\sim 1-2: 1$, lower than the value of $\sim 3-4: 1$ expected from XRB population synthesis models and observed in samples of nearby sources (Risaliti et al. 1999). This was explained by Treister et al. (2004) as due to the difficulty in obtaining spectroscopic identifications for obscured AGNs at significant redshifts, which are much fainter in the optical than the unobscured sources.

One significant problem of these deep multiwavelength surveys is caused by the effects of cosmic variance. For example, Gilli et al. (2005) found a factor of 2 difference between the correlation length for AGNs derived in the CDF-N and CDF-S fields, each covering $\sim 0.1 \mathrm{deg}^{2}$, and concluded that this difference could be explained by the effects of cosmic variance. In addition, significant overdensities and large-scale structures have been found in both fields (e.g., Gilli et al. 2003), which potentially affect the conclusions derived from each field individually. In order to address this problem, recently several wide-field deep surveys have been carried out. Examples of these surveys are the All-wavelength Extended Groth strip International Survey (AEGIS; Davis et al. 2007), the Cosmic Evolution Survey (COSMOS; Scoville et al. 2007; Koekemoer et al. 1997), and the Multiwavelength Survey by Yale-Chile (MUSYC; Gawiser et al. 2006a; Treister et al. 2007). The Extended Chandra Deep Field South (ECDF-S) is one of the four $30^{\prime} \times 30^{\prime}$ fields studied by MUSYC. This field is $\sim 3$ times larger than the existing CDF-N or CDF-S. Hence, while the effects of cosmic variance are reduced compared to the results of each of these deep fields, the uncertainty due to large-scale structure can still be relevant for our results. To minimize these effects, when possible, we combine 
in this work our data with available measurements in other fields.

Deep optical imaging has been obtained in the ECDF-S, mostly using the Wide-Field Imager (WFI) on the $2.2 \mathrm{~m}$ telescope at La Silla. Images in a narrowband filter centered at $500 \mathrm{~nm}$ were also obtained in this field in order to look for Lyman Alpha Emitters at $z \sim 3$ (Gawiser et al. 2006b). The ECDF-S imaging was made public by the ESO Deep Public Survey (Mignano et al. 2007), COMBO-17 (Wolf et al. 2004), and Garching-Bonn Deep Survey (GaBODS; Hildebrandt et al. 2006) teams. Deep near-IR coverage was obtained using the CTIO $4 \mathrm{~m}$ telescope with the Infrared Sideport Imager (ISPI), reaching a magnitude limit of $\sim 22(\mathrm{AB})$ in the $J H K_{s}$ bands ${ }^{10}$ (Taylor et al. 2009). In addition, the ECDF-S is the target of two Spitzer legacy surveys, the Spitzer Infrared Array Camera (IRAC)/MUSYC Public Legacy in ECDF-S (SIMPLE) and the Far-Infrared Deep Extragalactic Legacy Survey (FIDEL), which obtained deep mid-IR data from 3 to $70 \mu \mathrm{m}$. Most relevant for our work is the Chandra coverage of the ECDF-S, consisting of four ACIS-I pointings centered on the original CDF-S to a depth of $\simeq 230 \mathrm{ks}$ and covering an area of $\simeq 0.3 \mathrm{deg}^{2}$. A detailed description of these data was presented by Lehmer et al. (2005) and Virani et al. (2006). In this work, we use the Virani et al. (2006) catalog for the optical follow-up. A total of 651 sources are included in this catalog to a flux limit of $1.7 \times 10^{-16} \mathrm{erg}$ $\mathrm{cm}^{-2} \mathrm{~s}^{-1}$ in the $0.5-2 \mathrm{keV}$ band. These sources constitute the main sample of this work.

In this paper, we present the first results of an extensive spectroscopic effort aimed to identify a significant number of the Xray sources in the ECDF-S field. Optical spectroscopy allows us to measure redshifts, and hence luminosities, and also to provide an indication of the nature of the source, which can be compared with the observed multiwavelength properties. In addition, we will focus on the properties of the obscuring material by studying the mid-IR properties of these sources, and by measuring the fraction of obscured AGN and its dependence on parameters such as luminosity or redshift. In Section 2, we present the spectroscopic data and outline the reduction techniques used in this work, while in Section 3 the results are presented. In Section 4 , we take advantage of the exquisite multiwavelength coverage available on the ECDF-S to compare the X-ray, optical, and mid-IR properties of these sources. We also study the statistical properties of these sources in order to understand the evolution and physical conditions of the AGN population. The conclusions of our work are presented in Section 5. When required, we assume a $\Lambda$ cold dark matter $(\Lambda \mathrm{CDM})$ cosmology with $h_{0}=0.7, \Omega_{m}=0.3$, and $\Omega_{\Lambda}=0.7$, in agreement with the most recent cosmological observations (Spergel et al. 2007). All magnitudes in this paper are presented in the $\mathrm{AB}$ photometric system (Oke \& Gunn 1983).

\section{OPTICAL SPECTROSCOPY}

Sources targeted for spectroscopy were selected from the catalog of Chandra sources of Virani et al. (2006). We use this catalog instead of a similar one presented by Lehmer et al. (2005) since the former uses a more detailed rejection of high-background periods, thus eliminating spurious sources. The Virani et al. (2006) catalog consists of 651 X-ray detected sources, 587 detected at high significance (the primary catalog) and 64 found using a lower threshold (secondary catalog). The

\footnotetext{
${ }^{10}$ Optical and near-IR images and catalogs are public and can be obtained at http://www.astro.yale.edu/MUSYC.
}

X-ray sources were then matched to the MUSYC BVR optical catalog presented by Gawiser et al. (2006b) using a likelihood ratio technique, as described by Cardamone et al. (2008), using a reliability threshold of 0.6. The estimated number of false matches using this technique, calculated by randomly changing the X-ray positions, is $\sim 1 \%$. We found optical counterparts for 445 X-ray sources in the primary catalog $(76 \%)$ and 28 in the secondary catalog $(44 \%)$ down to a limiting magnitude of BVR $=27.1$. Using the Spitzer IRAC observations of the ECDF-S described in Section 2.5 and a similar likelihood ratio method, we found near-IR counterparts for 554 (94\%) X-ray sources in the primary catalog and $43(67 \%)$ in the secondary catalog. Hence, we are very confident that a very large fraction of the X-ray sources, greater than $90 \%$ in the primary and $\sim 70 \%$ in the secondary catalogs, are real.

While in principle all X-ray sources with optical counterparts were targeted for spectroscopy, we gave higher priority to the optically brightest sources, in order to increase the efficiency of our observations. In general, sources with $R<24$ had a higher priority, except for our VLT Visible Multi-Object Spectrograph (VIMOS) observations, in which an $R<25$ threshold was used. A total of 235 sources with $R<24$ and 292 with $R<25$ were targeted. As an experiment, for our Inamori Magellan Areal Camera and Spectrograph (IMACS) run of November we gave a higher priority to a group of $70 \mathrm{X}$-ray sources with bright optical counterparts $(R<24)$ and a hard X-ray spectrum, given by a hardness ratio (HR) lower than -0.2 . This was done in order to see if these sources have signs of obscuration in the optical spectrum as well, and to try to partially overcome the bias against obscured sources in identification studies based on optical spectroscopy. All these possible selection effects are considered in the analysis presented in Section 4.

\subsection{Magellan IMACS Data}

We used the IMACS wide-field spectrograph mounted on the Magellan I Baade telescope at Las Campanas Observatory. This camera provides a circular field of view (FOV) with a 27.2 radius in the $f / 2$ configuration. The pixel scale for this setup is $0^{\prime} \cdot 2$ pixel $^{-1}$. For all our observations we used the 300 lines $\mathrm{mm}^{-1}$ grism and slits of 1".2 width, except for the 2003 and 2006 October runs in which we used $1^{\prime \prime}$ slits. For our average seeing of $1^{\prime \prime}$, this translates into resolution elements of $8 \AA$ ( $6.5 \AA$ for the 2003 and 2006 October runs) except for the 2005 November run in which the average 0.' 6 seeing means a resolution element of $\sim 4 \AA$ for unresolved sources. While the wavelength coverage depends strongly on the position of the source in the mask, we required a minimum wavelength coverage of $4200-7000 \AA$, which maximizes the number of sources on a given mask while at the same time providing a broad wavelength coverage, which is important considering that the redshifts are not known a priori and can span a wide range. The log of our ECDF-S IMACS observations is presented in Table 1.

Typically each mask was observed for $5 \mathrm{hr}$. The goal was to reach a magnitude of $R \sim 24$, however in many cases, due to weather or technical problems, this was not possible. Hence, the efficiency, defined as the fraction of sources identified and with a measured redshift, differs significantly from mask to mask. The total number of sources on a typical mask is $\sim 80$ 100 , of which $15 \%-50 \%$ are X-ray sources. The large spread in the fraction of X-ray sources on a given mask reflects the fact that these sources were not always the main target of the observations, but "mask-fillers." The remaining targets include extragalactic sources like Ly- $\alpha$ emitters, Lyman break galaxies, 
Table 1

Log of Spectroscopic Observations

\begin{tabular}{|c|c|c|c|c|c|c|c|}
\hline Run & Instrument & Masks & Slit Width & Average Seeing & Total Sources & X-Ray Sources & Efficiency $^{\mathrm{a}}$ \\
\hline 2003 Oct $26-27$ & IMACS & 4 & $1^{\prime \prime} 0$ & $\sim 1^{\prime \prime}$ & 291 & 74 & $57 \%$ \\
\hline 2005 Feb 4-7 & IMACS & 2 & $1^{\prime \prime} .2$ & $0^{\prime \prime} 8$ & 180 & 64 & $57 \%$ \\
\hline 2005 Nov 2-3 & IMACS & 2 & $1^{\prime \prime} .2$ & $\sim 0.6$ & 194 & 29 & $66 \%$ \\
\hline 2006 Oct $25-27$ & IMACS & 3 & $1^{\prime \prime} 0$ & $0^{\prime \prime} .5-1^{\prime \prime} .5$ & 280 & 143 & $32 \%$ \\
\hline 2006 Nov 21-22 & IMACS & 1 & $1^{\prime \prime} .2$ & $\sim 0{ }^{\prime} .8$ & 109 & 17 & $20 \%$ \\
\hline 2007 Feb $18-20^{\mathrm{b}}$ & IMACS & 1 & $1^{\prime \prime} .2$ & $\sim 1^{\prime \prime}$ & 109 & 17 & $20 \%$ \\
\hline Period 78 & VIMOS & 4 & $1^{\prime \prime} 0$ & $1^{\prime \prime}$ & 283 & 96 & $54 \%$ \\
\hline
\end{tabular}

Notes.

a Defined as the fraction of X-ray sources identified.

b The mask from the 2006 November run was reobserved in order to obtain $\sim 5 \mathrm{hr}$ of integration time.

and galactic white and brown dwarf stars selected by their optical colors from the MUSYC catalogs. The properties of the general sources targeted with IMACS will be presented in a following paper (P. Lira et al. 2009, in preparation).

\subsection{VLT VIMOS Data}

The VIMOS (Le Fèvre et al. 2003) spectrograph mounted at the Nasmyth focus B of UT3 was also used to obtain optical spectroscopy for the X-ray sources in the ECDF-S. This instrument consists of four cameras of $7^{\prime} \times 8^{\prime}$ each, hence providing a total FOV of $15^{\prime} \times 16^{\prime}$, with a pixel scale of $0^{\prime \prime} 2$ pixel $^{-1}$. We used the MR grism together with the OS-blue filter in order to block the contamination from higher orders. In this setup, the wavelength coverage is $4000-6700 \AA$, very similar to the coverage of the IMACS observations. This coverage minimizes the effects of fringing at red wavelengths and takes advantage of the wavelength region of maximum efficiency of the VIMOS camera. The MR grism provides a dispersion of $2.5 \AA$ pixel $^{-1}$, which combined with our $1^{\prime \prime}$ wide slits corresponds to a resolution element of $12.5 \AA$. All the VIMOS observations were carried out in service mode by the Paranal staff. The required conditions were seeing better than $1^{\prime \prime}$, clear sky and fractional lunar illumination smaller than $20 \%$ at a minimum distance of $30^{\circ}$. Due to the lack of an Atmospheric Dispersion Corrector in VIMOS and in order to minimize the effects of instrument flexures, observations were carried out at a maximum hour angle of $\pm 2 \mathrm{hr}$.

Four pointings were used to completely cover the ECDF-S $30^{\prime} \times 30^{\prime}$ field. Each mask was observed for $3 \mathrm{hr}$ and 18 minutes, which corresponds to a total execution time of $6 \mathrm{hr}$ including overheads. According to the exposure time calculator this should allow us to significantly detect sources up to $R \sim 24.5$. A total of 283 sources were targeted, excluding alignment stars, 96 of them X-ray sources.

\subsection{Additional Observations}

Since the ECDF-S is the target of many deep multiwavelength observations, it is not surprising that many spectroscopic follow-up programs have been carried out in this field. In order to take advantage of existing observations of these X-ray sources, we did an extensive literature search. In particular, we used the Master Compilation of GOODS/CDF-S spectroscopy. ${ }^{11} \mathrm{We}$ matched this spectroscopic catalog to our MUSYC optical catalog using a search radius of $0{ }^{\prime \prime} 7$. Then, we used the MUSYCChandra matched catalog described before and found spectroscopic identifications for the X-ray sources in the observations

\footnotetext{
11 Available at

http://www.eso.org/science/goods/spectroscopy/CDFS_Mastercat/.
}

reported by Vanzella et al. (2005, 2006, 2008), Le Fèvre et al. (2004), Szokoly et al. (2004), and Croom et al. (2001). In total, existing identifications of $55 \mathrm{X}$-ray sources were added to our sample.

We also searched for existing unpublished spectroscopic observations in the ESO-VLT archive. ${ }^{12}$ We focused on VIMOS, given the large area covered by this camera and that most of the existing FORS2 observations were already incorporated in the master compilation described above. There are several VIMOS observations of the ECDF-S on the ESO archive; however, of particular relevance for us was the program of Bergeron et al. (program ID 072.A-0139), for which $23 \mathrm{hr}$ were granted in order to obtain spectroscopy of the $\mathrm{X}$-ray sources detected by $X M M$. The area covered by $X M M$ is well matched to the ECDF-S Chandra observations, so it is reasonable that a significant number of our Chandra sources were identified as part of this program. Three fields covered by the Bergeron program overlap with the ECDF-S. These fields were observed using both the MR and LR-Blue grisms with the GG475 and OS-Blue filters, respectively. We looked for counterparts of our Chandra sources using a search radius of $2^{\prime \prime}$, which was chosen in order to account for the $X M M$ positional uncertainty but at the same time avoid misidentifications. In total, 36 sources in our Chandra catalog were observed as part of the Bergeron program; 28 of them were already targeted as part of our IMACS and VIMOS program, but only 11 of them were successfully identified, so potentially identifications for 25 new sources could be obtained from the archival data.

\subsection{Data Reduction}

The IMACS data were reduced using the Carnegie Observatories System for MultiObject Spectroscopy (COSMOS) package $^{13}$ developed by Gus Oemler. This software was specifically designed to reduce IMACS data, in particular handling the challenge of having spectra spread over several CCDs. We followed the standard reduction cookbook, which consists of the following steps: construction of the spectral map using arc frames, bias-subtraction and flat fielding of science data and sky subtraction. We used COSMOS to generate two-dimensional sky-subtracted wavelength-calibrated spectra of each source. We then used IRAF's apall task in order to extract the onedimensional spectrum.

To reduce the VIMOS data we used the ESO VIMOS pipeline version 2.1.6. ${ }^{14}$ In this case, all the spectra for a given quadrant

\footnotetext{
12 The ESO archive can be found at http://archive.eso.org.

13 This software can be obtained from http://www.ociw.edu/Code/cosmos.

14 This package can be obtained from

http://www.eso.org/projects/dfs/dfs-shared/web/vlt/vlt-instrument-pipelines.html.
} 
are located on one CCD, so the data reduction is significantly simpler than for IMACS data. However, the basic reduction steps are very similar to the IMACS reduction. The same procedure was used to reduce both the archival observations and our proprietary data. In the case of the VIMOS data from our program, for each individual observation of $\sim 33$ minutes, the corresponding night-time arc was used to calculate the spectral map, in order to account for the effects of instrument flexures. In addition, observations of standard stars on the same night were used to calculate the effective response as a function of wavelength for these observations.

\subsection{Spitzer Data}

In order to study the IR to X-ray ratio for our sample of sources with measured spectroscopic redshifts in the ECDF-S we took advantage of the deep Spitzer images available in this field. At the shortest wavelengths, the central CDF-S region $\left(10^{\prime} \times 16^{\prime}\right)$ was observed by IRAC (Fazio et al. 2004) as part of the GOODS survey to flux limits of $0.13,0.22,1.45$, and $1.61 \mu \mathrm{Jy}$ in the 3.6, 4.5, 5.7, and $8 \mu \mathrm{m}$ band, respectively. Source matching and basic IR properties were reported by Treister et al. (2006). In addition, the whole ECDF-S field was covered by IRAC as part of the SIMPLE survey ${ }^{15}$ (M. Damen et al. 2009, in preparation). The flux limits for the SIMPLE observations are $0.76,0.4,5.8$, and $3.6 \mu \mathrm{Jy}$ in the 3.6, 4.5 and 5.7, and $8 \mu \mathrm{m}$ bands, respectively, thus $\sim 3-5$ times shallower than the GOODS observations. The matched catalog of the ECDF-S Xray sources to the SIMPLE images used here was presented and described in detail by Cardamone et al. (2008).

At longer wavelengths, we use the available Multiband Imaging Photometer for Spitzer (MIPS; Rieke et al. 2004) observations at $24 \mu \mathrm{m}$. In the central region, GOODS provide deep images to a flux limit of $\sim 25 \mu \mathrm{Jy}$. A catalog ${ }^{16}$ based on pointspread function (PSF)-fitting magnitudes, described by Treister et al. (2006) and R. Chary et al. (2009, in preparation), was matched to the X-ray sources in the GOODS region using the IRAC-derived positions.

Additional data at $24 \mu \mathrm{m}$ covering the whole ECDF-S field were taken as part of the FIDEL survey. Unfortunately, at the moment only the reduced images were made available by the FIDEL group. ${ }^{17}$ In order to produce our own catalog based on the reduced FIDEL images we used the Mosaicking and Point-Source Extraction (MOPEX; Makovoz \& Marleau 2005) package, provided by the Spitzer Science Center. Briefly, we did a first-pass extraction using the default point response function (PRF) included in MOPEX in order to obtain a preliminary catalog of the brightest sources. These sources were then used to construct a customized PRF, based directly in the FIDEL observations. Finally, using this PRF we performed a second extraction and measured PRF-fitting flux densities for the detected sources. This procedure is described in more detail by Makovoz \& Marleau (2005). In order to verify the accuracy of our derived fluxes in Figure 1 we compare the FIDEL and GOODS sources for the 829 overlapping sources with fluxes higher than $60 \mu \mathrm{Jy}$ in order to ensure a reliable detection in

\footnotetext{
15 Further information about this survey, catalogs and images can be found at the Web site http://www.astro.yale.edu/dokkum/simple/.

16 More details about this catalog and data-reduction procedures can be found at the Web site http://data.spitzer.caltech.edu/popular/goods/Documents/ goods_dataproducts.html.

17 The reduced images and more details about the survey and reduction techniques can be found at

http://data.spitzer.caltech.edu/popular/fidel/2007_sep17/fidel_dr2.html.
}

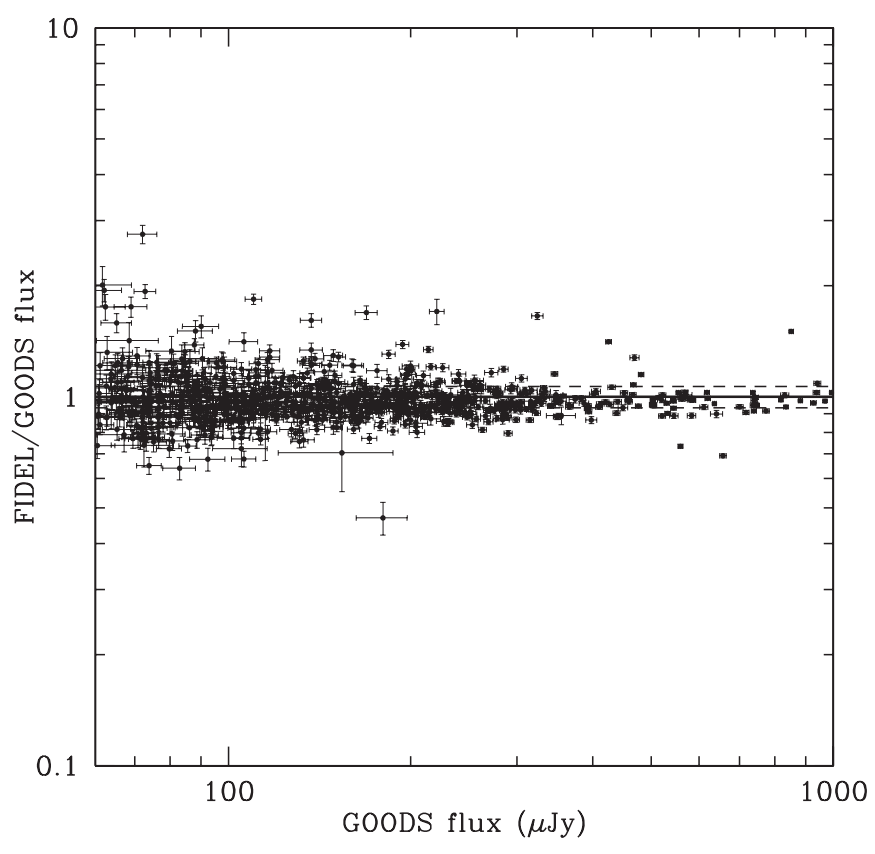

Figure 1. Ratio of PSF-fitting $24 \mu \mathrm{m}$ fluxes derived from the FIDEL images and GOODS catalogs as a function of GOODS flux for the sources significantly detected by both surveys. The median value for the flux ratio is 0.97 , with a standard deviation of $7 \%$, well within the measurement errors, showing that the two studies produced consistent results.

the FIDEL image. There is a very good agreement between the FIDEL and GOODS fluxes. The median ratio of FIDEL to GOODS flux is 0.97 with a standard deviation of $7 \%$. Given the very small systematic offset between the FIDEL and GOODS fluxes, well within the measurement errors, no correction to the FIDEL fluxes was applied. The FIDEL catalog was matched to the ECDF-S using the maximum likelihood procedure described by Cardamone et al. (2008).

\section{RESULTS}

A total of 339 X-ray sources in the Virani et al. (2006) catalog were targeted for spectroscopy using the VIMOS and IMACS spectrographs and data from the literature. For 186 of them, we were able to identify the nature of the source and measure the redshift, giving an average efficiency of 55\%. Of those, 180 were detected in the high-significance primary X-ray catalog. The basic X-ray, optical, and spectroscopic properties of these sources are presented in Table 2 . The $R$-band magnitude distributions for all the X-ray sources, the sources targeted for spectroscopy and those with spectroscopic identification are presented in Figure 2. The fraction of sources that are successfully identified depends strongly on the observing conditions, achieved exposure time, etc. (Table 1). At the same time, this fraction always decreases strongly toward fainter magnitudes (Figure 2). In our analysis, we model this dependence using a very simple linear fit, with a constant value of $65 \%$ to $R=22$ and then decreasing linearly to $0 \%$ at $R=26.5$. The resulting fit is shown in the upper panel of Figure 2.

The redshift distribution for our spectroscopically identified sources is presented in Figure 3. The vast majority of unobscured sources (that present broad emission lines, see discussion below) are at $z>1$, with an average redshift of 2.22 and a median of 2.12. In contrast, obscured AGN and X-ray emitting galaxies are preferentially located at $z<1$. For the total sample, the average redshift is 1.18 , with a median of 0.75 , in good agreement with 
Table 2

X-Ray, Optical, and Mid-IR Properties of the X-Ray Sources in the ECDF-S

\begin{tabular}{|c|c|c|c|c|c|c|c|c|c|c|c|c|c|c|c|c|}
\hline \multirow[t]{2}{*}{$\mathrm{ID}^{\mathrm{a}}$} & \multicolumn{2}{|c|}{ X-Ray Flux (erg $\left.\mathrm{cm}^{2} \mathrm{~s}^{-1}\right)$} & \multicolumn{3}{|c|}{ Hardness Ratio } & \multirow[t]{2}{*}{ Redshift } & \multirow[t]{2}{*}{ Instrument } & \multirow{2}{*}{ Class. $^{\mathrm{d}}$} & \multirow{2}{*}{$\begin{array}{c}\log (\text { X-Ray Lum.) } \\
\left(\operatorname{erg~s}^{-1}\right)\end{array}$} & \multicolumn{3}{|c|}{$24 \mu \mathrm{m}(\mathrm{mJy})$} & \multicolumn{3}{|c|}{$\Gamma$} & \multirow{2}{*}{$\begin{array}{c}N_{H} \\
\left(\mathrm{~cm}^{-2}\right)\end{array}$} \\
\hline & Soft $^{b}$ & $\operatorname{Hard}^{\mathrm{c}}$ & Value & Upper & Lower & & & & & Flux & Error & Source $^{\mathrm{e}}$ & Value & Upper & Lower & \\
\hline 2 & $2.040 \times 10^{-15}$ & $4.690 \times 10^{-15}$ & 1.000 & 0.000 & 0.000 & $9.990^{\mathrm{f}}$ & IMACS & UNK & & $\ldots$ & & - & $\ldots$ & & & \\
\hline 3 & $2.670 \times 10^{-15}$ & $6.620 \times 10^{-15}$ & -1.000 & 0.000 & 0.000 & 9.990 & IMACS & UNK & $\ldots$ & 183.50 & 3.50 & $\mathrm{~F}$ & 1.900 & & $\ldots$ & 20.843 \\
\hline 4 & $3.650 \times 10^{-14}$ & $6.590 \times 10^{-14}$ & -0.354 & 0.030 & 0.031 & 2.011 & IMACS & OAGN & 45.273 & 686.40 & 4.71 & $\mathrm{~F}$ & 1.836 & 0.123 & 0.117 & 20.941 \\
\hline 6 & $6.760 \times 10^{-15}$ & $8.590 \times 10^{-15}$ & -0.498 & 0.069 & 0.075 & 3.031 & VIMOS & UAGN & 44.821 & 273.30 & 1.53 & $\mathrm{~F}$ & 2.045 & 0.402 & 0.347 & 21.087 \\
\hline 7 & $1.260 \times 10^{-15}$ & $4.300 \times 10^{-15}$ & -0.054 & 0.126 & 0.146 & 0.289 & IMACS & ELG & 42.044 & $\ldots$ & $\ldots$ & - & $\ldots$ & $\ldots$ & $\ldots$ & $\ldots$ \\
\hline
\end{tabular}

Notes.

${ }^{a}$ X-ray ID number from the catalog of Virani et al. (2006).

b $0.5-2 \mathrm{keV}$.

c $2-8 \mathrm{keV}$

d UNK: unkown; OAGN: obscured AGN; UAGN: unobuscred AGN; ELG: emission line galaxy; ALG: absorption line galaxy; STAR: star.

e Survey in which the $24 \mu \mathrm{m}$ source is identified. F: FIDEL; G: GOODS.

${ }^{\mathrm{f}} \mathrm{A} z=9.990$ corresponds to a targeted source for which the redshift could not be measured.

(This table is available in its entirety in a machine-readable form in the online journal. A portion is shown here for guidance regarding its form and content.)

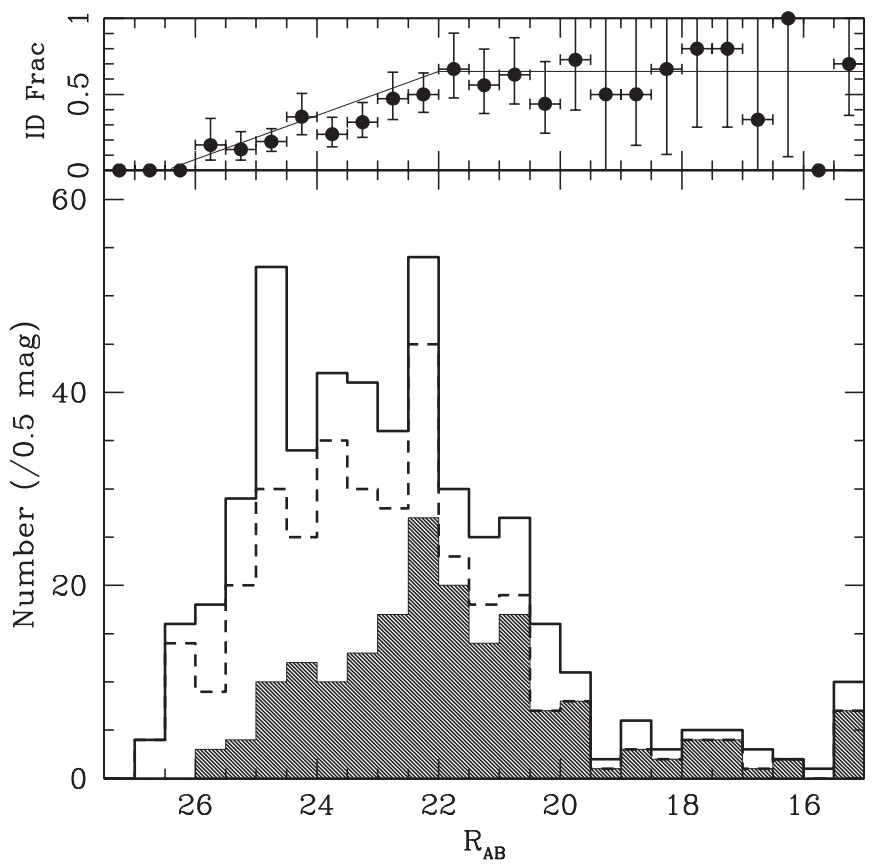

Figure 2. Bottom panel: distribution of $R$-band magnitude for all $\mathrm{X}$-ray sources with optical counterpart in the MUSYC images (solid line), sources targeted for spectroscopy (dashed), and identified sources (hatched histogram). Top panel: fraction of identified to total X-ray sources as a function of $R$-band magnitude. The thin solid line shows the fit to the data points, used in Section 4 . The fraction of identified sources is $\sim 65 \%$ at $R<2$, and decreases toward fainter optical fluxes.

the results found in both CDFs (Szokoly et al. 2004; Barger et al. 2003; Gilli et al. 2005). The highest redshift source in our sample is a broad-line AGN, XID 213, at $z=4.48$.

In the upper panel of Figure 3, we use a narrower bin width $(\Delta z=0.02)$, in order to search for structures in redshift space. The two main structures, "walls," previously detected in the CDF-S proper at $z=0.67$ and $z=0.73$ (Gilli et al. 2003; Adami et al. 2005) are also easily found in our sample. In addition, we found excesses of sources at $z=0.15,0.29$, and 0.53. In Figure 4, we present the spatial distribution of all the identified sources, with the position of the sources in those redshift structures highlighted. For $z=0.15,0.29$, and 0.53 , the $30^{\prime} \times 30^{\prime}$ ECDF-S field corresponds to a comoving area of $4.6 \times 4.6,7.7 \times 7.7$, and $11.2 \times 11.2 \mathrm{Mpc}^{2}$, respectively. Given

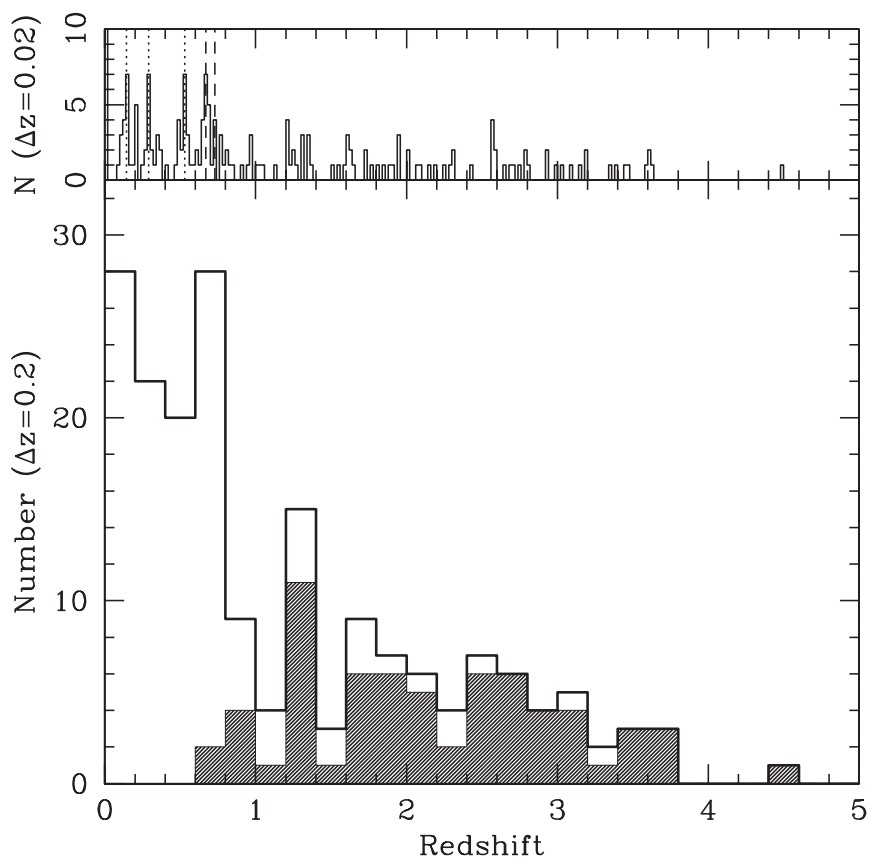

Figure 3. Bottom panel: redshift distribution for all X-ray sources with spectroscopic identification (solid line) and sources classified as unobscured AGNs only (hatched histogram) in bins of $\Delta z=0.2$. Top panel: redshift distribution for all sources in bins of $\Delta z=0.02$, in order to identify overdensities. The dashed lines mark the previously known structures in this field at $z=0.67$ and 0.73 , while the dotted lines show other suspected overdensities at $z=0.15$, 0.29 , and 0.53 .

that all the redshift groups span almost the whole FOV, these structures are much larger than the compact structures reported by Adami et al. (2005). In order to estimate the probability of having seven sources in one $\Delta z=0.02$ bin we assume that the redshift distribution follows a Poisson distribution. The average number of sources in a $\Delta z=0.02$ bin is $\simeq 2$. Hence, the probability of having seven sources in one bin randomly is $\simeq 0.3 \%$. Therefore, we can conclude that these groups in redshift space should correspond to real physical associations.

Sources were mainly classified according to their optical spectroscopy characteristics. Our primary division comes from the presence or absence of broad emission lines. The threshold for unobscured sources was set at $\Delta v>1000 \mathrm{~km} \mathrm{~s}^{-1}$, since Barger et al. (2005) showed that the vast majority of the 


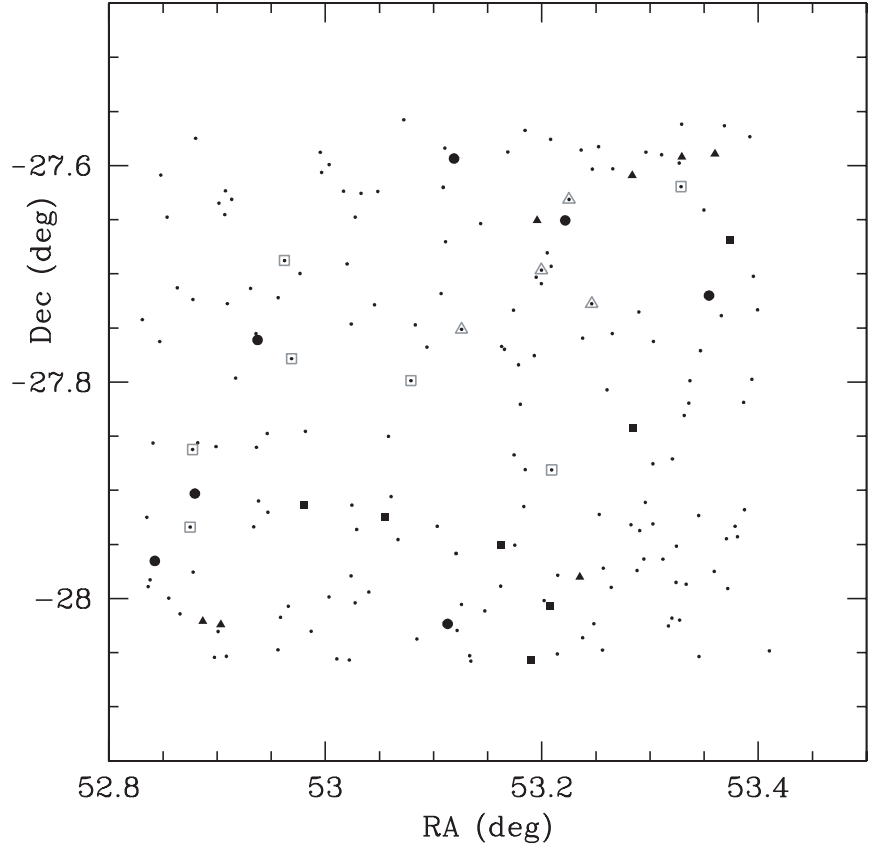

Figure 4. Small solid circles: location of identified sources in the ECDF-S field. Other symbols mark the sources in the identified overdensities: solid triangles: $z=0.15$, solid squares: $z=0.29$, large solid circles: $z=0.53$, open squares: $z=$ 0.67 , open triangles: $z=0.73$. As can be seen, sources in the groups in redshift space span almost the whole studied area.

non-X-ray sources have line widths smaller than this value, while sources with broad lines have also soft X-ray spectra, consistent with being dominated by an unobscured AGN. We then used the hard (2-8 keV) X-ray luminosity to separate normal galaxies with X-ray emission from AGN-dominated sources. Specifically, we assumed a conservative and typical threshold of $L_{X}=10^{42} \mathrm{erg} \mathrm{s}^{-1}$, higher than the highest level of $\mathrm{X}$-ray activity observed in star-forming galaxies (e.g., Lira et al. 2002).

The X-ray spectral shape can be used to obtain an alternative source classification. In particular, the HR, defined as the ratio between the difference of the hard band (typically $2-8 \mathrm{keV}$ for Chandra observations) and soft band (0.5-2 keV) count rates and their sum is used when the number of detected counts is not enough to perform X-ray spectral fitting. Figure 5 shows the HR as a function of luminosity for the 186 sources with spectroscopic identifications presented in this paper. As can be seen, sources with hard X-ray spectrum (positive HR) tend to be also optically classified as obscured sources in the optical, while sources that present broad emission lines have in general a soft $\mathrm{X}$-ray spectrum (negative HR). It is also interesting that most of the unobscured sources, in both X-rays and optical wavebands, have a high X-ray luminosity $\left(L_{X}>10^{43} \mathrm{erg} \mathrm{s}^{-1}\right)$, while most obscured sources are fainter in X-rays. These trends will be studied in further detail in the following section.

While the HR can be used as a rough indication of the $\mathrm{X}$ ray spectral shape, for the brighter X-ray sources it is possible to perform more accurate spectral fitting. In the case of the ECDF-S, a total of 184 sources have more than 80 counts detected in the $0.5-8 \mathrm{keV}$ Chandra band, making spectral fitting possible. We used a modified version of the Yaxx $^{18}$ software to extract the spectrum of each high-significance source. For sources with more than 200 background-subtracted counts and measured redshifts, 72 in the ECDF-S, we simultaneously fitted

\footnotetext{
18 Available at http://cxc.harvard.edu/contrib/yaxx/.
}

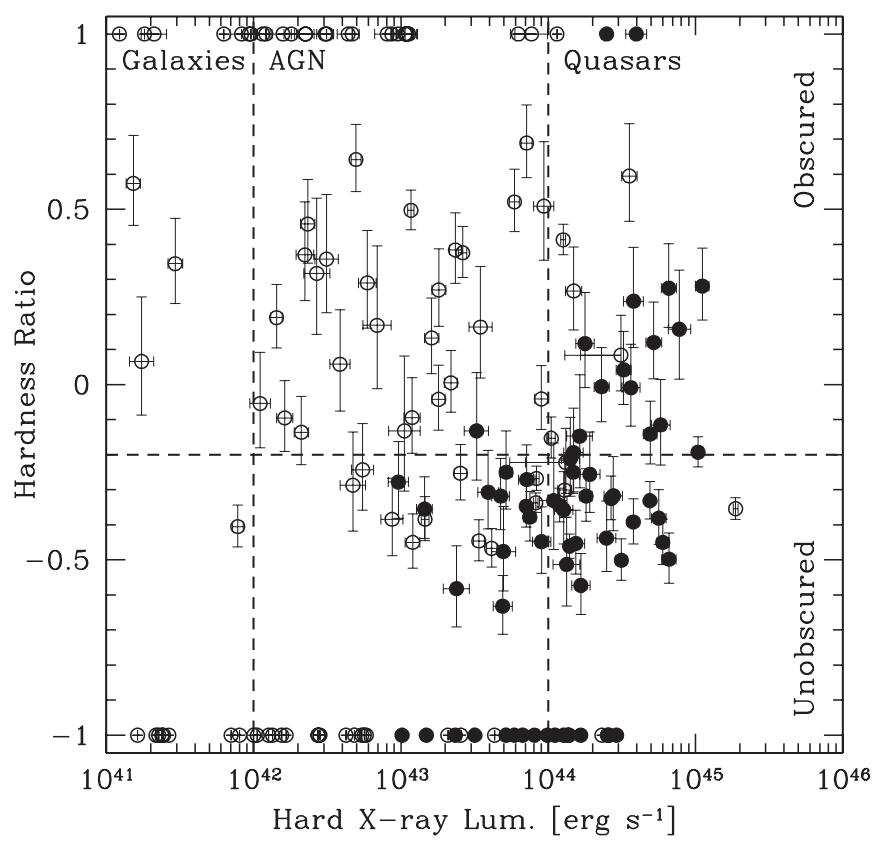

Figure 5. HR, defined as $(H-S) /(H+S)$ where $H$ and $S$ are the hard $(2-8 \mathrm{keV})$ and soft $(0.5-2 \mathrm{keV}) \mathrm{X}$-ray count rates, as a function of hard X-ray luminosity for sources optically classified as obscured (open circles) and unobscured (filled circles) AGNs. Vertical dashed lines show the typical separation for normal galaxies $\left(L_{X}<10^{42} \mathrm{erg} \mathrm{s}^{-1}\right)$, Seyfert galaxies $\left(L_{X}=10^{42-44} \mathrm{erg} \mathrm{s}^{-1}\right)$, and quasars $\left(L_{X}>10^{44} \mathrm{erg} \mathrm{s}^{-1}\right)$. The dashed horizontal line shows the HR for a source with $\Gamma=1.9$ and $N_{\mathrm{H}}=10^{22} \mathrm{~cm}^{-2}$ at $z<0.5$. Most sources classified as unobscured AGNs are clustered in the high luminosity and small HR region, while obscured AGNs have in general lower luminosities and higher HR.

a power-law spectrum and photoelectric absorption, with three free parameters: slope of the power law $(\Gamma)$, normalization, and observed neutral hydrogen column density $\left(N_{\mathrm{H}}\right)$. In all cases, we included the Galactic absorption, assuming an average $N_{\mathrm{H}}$ of $6.8 \times 10^{19} \mathrm{~cm}^{-2}$ for the ECDF-S, as measured by the survey of Galactic HI of Kalberla et al. (2005). For sources with less than 200 but more than 80 counts, it was not possible to perform such detailed fitting, so we fixed the slope of the power law to a value of $\Gamma=1.9$, very similar to the average value for the sources with a fitted slope $(\Gamma=1.95)$, which also corresponds to the typical X-ray spectrum for unobscured AGNs (Nandra \& Pounds 1994; Nandra et al. 1997 and others). In both cases, the $N_{\mathrm{H}}$ value was derived in the observed frame, in order to keep the $\mathrm{X}$-ray spectral fitting independent of the redshift measurements. In order to calculate the conversion from observed to intrinsic $N_{\mathrm{H}}$ we used the Xspec version 12.4.0 software (Arnaud 1996) to simulate an X-ray spectrum with $\Gamma=1.9$ as observed at the Chandra aimpoint with ACIS-I, added absorption at varying redshifts from 0 to 4 and measured the fitted $N_{\mathrm{H}}$ value in the observed frame. We obtained an empirical relation of $N_{\mathrm{H}}$ (intr) $=(1+z)^{2.65} N_{\mathrm{H}}$ (obs), consistent with the conversion assumed in the past for Chandra data (e.g., Bauer et al. 2004). Furthermore, we checked that the derived relation is roughly independent of the assumed slope of the power-law spectrum. Hence, we are very confident that our conclusions are not affected by our choice of measuring absorption in the observed frame.

The conversion from observed to intrinsic $N_{\mathrm{H}}$ can thus only be done for sources with a measured redshift. We have spectroscopic redshifts, given in Table 2, for a total of 80 sources with fitted $N_{\mathrm{H}}$. However, in order to increase our sample we used the good quality photometric redshifts derived by the COMBO17 survey, which provides redshifts accurate to $\delta z /(1+z) \sim 10 \%$ for sources with $R<24$ (Wolf et al. 2004). This uncertainty in 


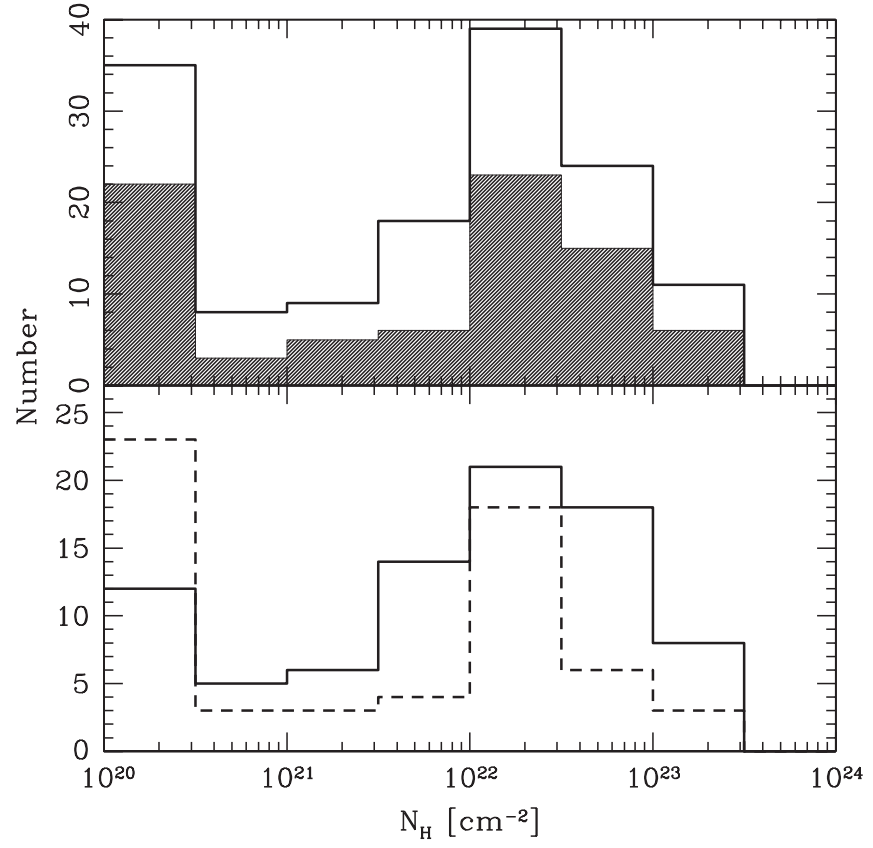

Figure 6. Top panel: distribution of $N_{\mathrm{H}}$ for all the sources with either spectroscopic or photometric redshift and more than 80 counts in X-rays. The hatched histogram shows the distribution for sources with spectroscopic redshifts only. According to a $\mathrm{KS}$ test, the distributions are entirely consistent with being drawn from the same parent distribution. Bottom panel: $N_{\mathrm{H}}$ distribution for sources optically classified as obscured (solid histogram) and unobscured (dashed histogram). While X-ray obscuration is present in unobscured AGNs, most of them have very small $N_{\mathrm{H}}$ values. Similarly, sources classified as obscured AGNs in the optical have in general higher $N_{\mathrm{H}}$ values. However, in both cases significant scatter is present.

redshift translates into an uncertainty in the derived intrinsic $N_{\mathrm{H}}$ of $\delta \log N_{\mathrm{H}} \simeq 0.12$, smaller than the typical errors in the fitted observed $N_{\mathrm{H}}$ or the bin size in our $N_{\mathrm{H}}$ distribution (Figure 6). Hence, it is acceptable to use the COMBO-17 photometric redshifts in order to convert observed $N_{\mathrm{H}}$ into intrinsic values, thus increasing our sample to 144 sources. To confirm that the use of COMBO-17 redshifts does not bias our conclusions, we performed a Kolmogorov-Smirnov (KS) test and found that the hypothesis that both distributions were drawn from the same parent distribution can only be dismissed at the $0.02 \%$ confidence level.

\section{DISCUSSION}

\subsection{X-Ray/Optical Classification}

The bottom panel of Figure 6 shows the distribution of intrinsic $N_{\mathrm{H}}$ separately for sources classified as obscured and unobscured AGNs based only on optical spectroscopy. While the average value of $N_{\mathrm{H}}$ is larger for obscured than unobscured sources, $8 \times 10^{21} \mathrm{~cm}^{-2}$ versus $2 \times 10^{21} \mathrm{~cm}^{-2}$, the difference is not very large. Performing a KS test to these two distributions rules out the hypothesis that they were drawn from the same parent distribution with a $98.5 \%$ confidence, leaving a small but not completely negligible probability. Since they are both indications of the X-ray spectral shape, there is a close relationship between the HR and derived $N_{\mathrm{H}}$ values. As can be seen in Figure 7, for modest column densities, $N_{\mathrm{H}} \leqslant 10^{22.5}$ the $N_{\mathrm{H}}$ values are very sensitive to small changes in the number of detected soft X-ray photons, in particular for high redshift sources. This implies that there can be large errors in the derived $N_{\mathrm{H}}$ values, in particular for unobscured sources at high redshift. The im-

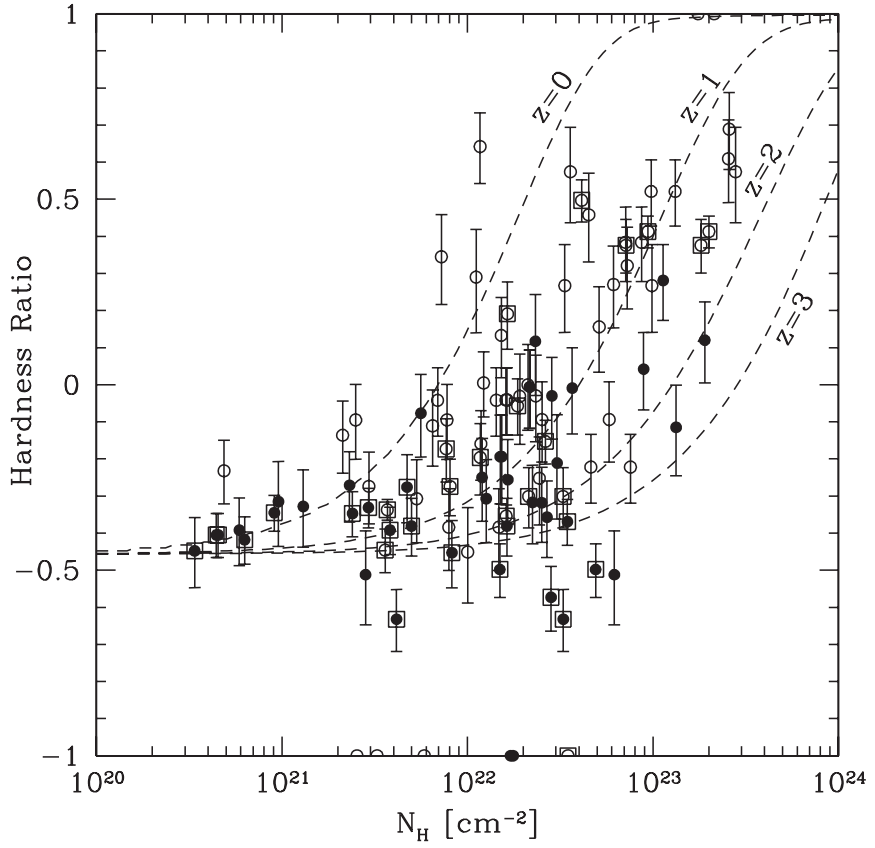

Figure 7. Neutral hydrogen column density, $N_{\mathrm{H}}$, as a function of HR. Sources optically classified as unobscured AGNs are shown by solid circles, while obscured AGNs are marked by open circles. Sources with more than 200 X-ray counts detected are enclosed in squares. The dashed lines show the relation between $N_{\mathrm{H}}$ and HR for a power-law source with $\Gamma=1.9$ at $z=0,1,2$, and 3 . At $N_{\mathrm{H}} \leqslant 10^{22.5}$, and in particular at high redshift, the $N_{\mathrm{H}}$ measurements are very sensitive to small changes in HR. This can explain the presence of optically unobscured sources with high $N_{\mathrm{H}}$ values.

plications of this potential bias in our analysis will be further discussed below.

As was shown in Figures 5 and 7, the optical and Xray classifications do not always agree. In order to further investigate this effect, in the left panel of Figure 8 we show the intrinsic $N_{\mathrm{H}}$ versus hard X-ray luminosity, used as a tracer of the intrinsic AGN luminosity. For this figure we also added $335 \mathrm{X}$-ray sources detected in the $1 \mathrm{Ms}$. CDF-S observations with accurate $N_{\mathrm{H}}$ measurements based on spectral fitting were presented by Tozzi et al. (2006). Redshifts for those sources, either photometric or spectroscopic, were compiled by Zheng et al. (2004). This figure shows two interesting effects. At low luminosities, $L_{X}<10^{43} \mathrm{erg} \mathrm{s}^{-1}$, a large fraction of the sources optically classified as obscured AGNs have intrinsic $N_{\mathrm{H}}$ values lower than $10^{22} \mathrm{~cm}^{-2}$. This can be explained by the effects of dilution of the AGN light by the host galaxy (e.g., Moran et al. 2002; Cardamone et al. 2007), which lowers the equivalent width of the broad emission lines because of the relatively high continuum from the host galaxy, thus making a source to be erroneously classified as obscured AGNs in the optical. A second effect appears at high luminosity: sources optically classified as unobscured AGNs have intrinsic $N_{\mathrm{H}}$ values higher than $10^{22} \mathrm{~cm}^{-2}$. A possible explanation for this effect can be found in the right panel of Figure 8 , where we can see that the sources with discrepant X-ray/optical classifications are predominantly located at $z>2.5$. Given the strong redshift dependence of the accuracy of the $N_{\mathrm{H}}$ determinations, sources with very small observed $N_{\mathrm{H}}$ values, which can be in general explained by measurement errors, will have a high intrinsic $N_{\mathrm{H}}$ values if the source is located at high redshift. At high redshift, the Chandra observations trace emission at higher energy in the rest frame, thus making it much harder to detect the photoelectric absorption cutoff, critical for measuring $N_{\mathrm{H}}$. This effect was 

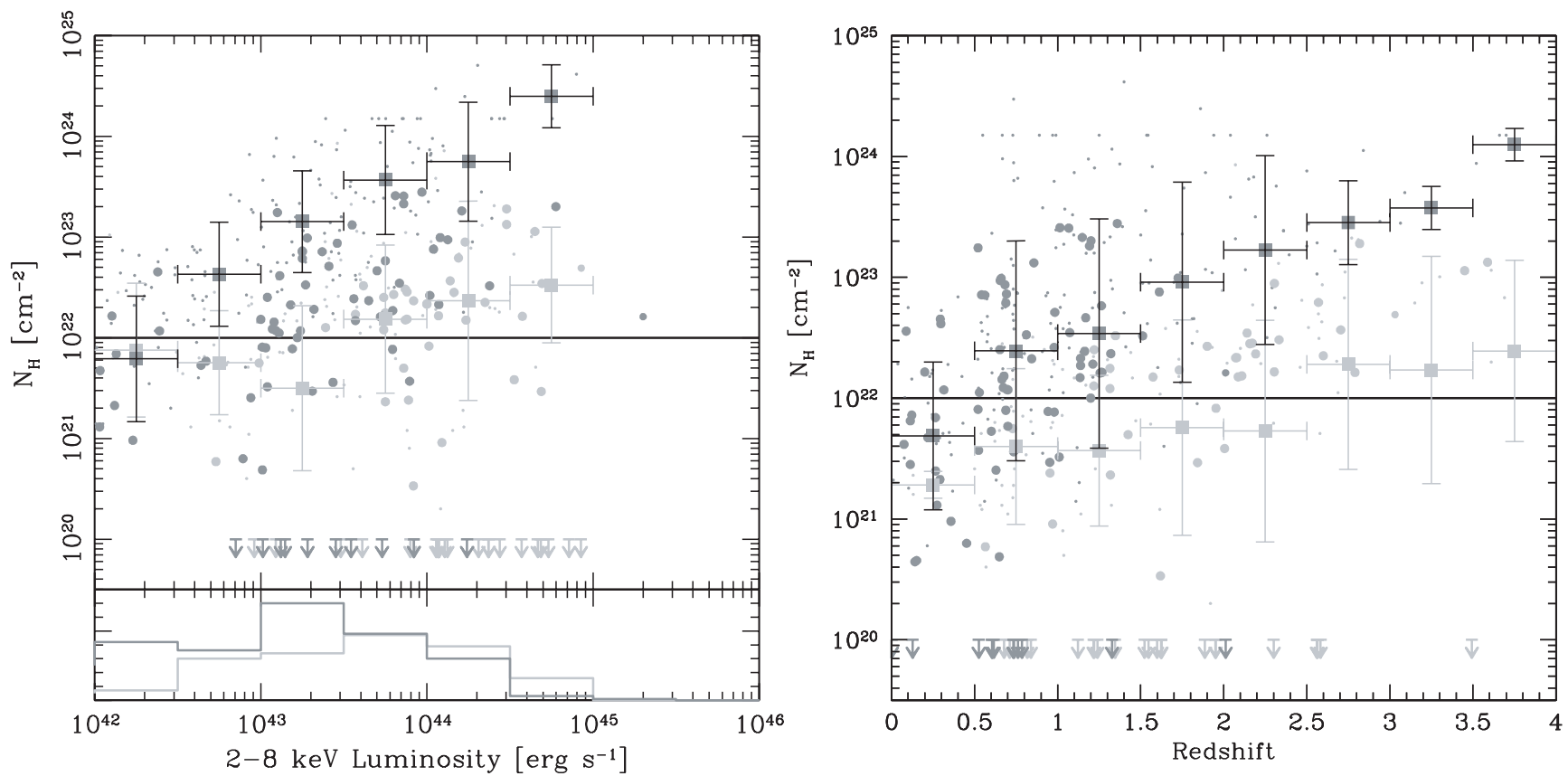

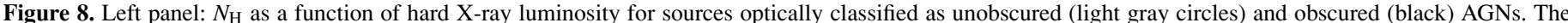

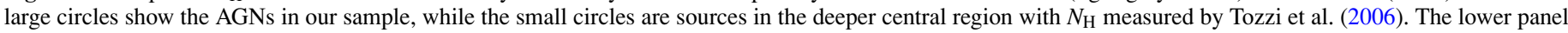

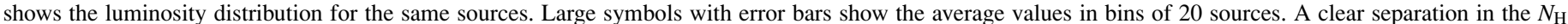

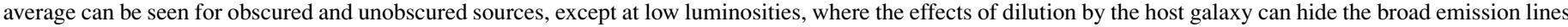

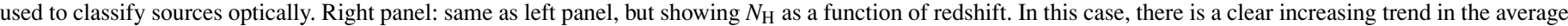

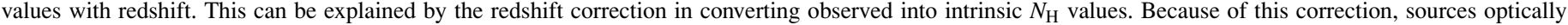
classified as unobscured at high redshift, can appear to have large $N_{\mathrm{H}}$ values, as discussed in the text.

already studied and simulated by Akylas et al. (2006), who found that at high redshift the derived column densities are systematically overestimated. According to the results of Akylas et al. (2006), $N_{\mathrm{H}}$ measurements are systematically overestimated by $\sim 50 \%$ at $z \sim 2.5$ and $\sim 20 \%$ at $z \sim 1.5$.

An obvious conclusion of this analysis is that no classification method is perfect and can be used for all sources. An early attempt to use a combined X-ray/optical classification was performed by Szokoly et al. (2004). In their work, they used a separation between obscured and unobscured sources at a HR of -0.2. A similar approach was used by Hasinger et al. (2005) to select unobscured AGNs from an X-ray-selected sample. This threshold is shown in Figure 5 for the sources with spectroscopic identification in the ECDF-S. A HR of -0.2 corresponds to an intrinsic $N_{\mathrm{H}}$ of $10^{22} \mathrm{~cm}^{-2}$ at $z=0.5$, assuming $\Gamma=1.9$, while sources with this $N_{\mathrm{H}}$ at lower redshift will have a higher (more positive) value. So, a threshold of -0.2 in HR to separate obscured and unobscured AGNs is appropriate at $z<$ 0.5 . However, as is clear from Figure 8 , the effects of dilution by the host galaxy are important only for low luminosity sources, which are predominantly located at low redshift. Hence, we propose the following classification scheme. For sources at $z<$ 0.5 , we will separate obscured and unobscured sources at $\mathrm{HR}=$ -0.2 , regardless of their optical properties, while for sources at higher redshifts we will use only the optical classification scheme, as $N_{\mathrm{H}}$ measurements are biased at high redshift.

In Figure 9, we show the measured $N_{\mathrm{H}}$ values as a function of luminosity and redshift for the new classification scheme. As expected, changes are relevant only for sources with low luminosities at low redshifts. With an optical classification only, the average $N_{\mathrm{H}}$ for obscured sources at $z<0.5$ is $4.8 \times 10^{21}$ $\mathrm{cm}^{-2}$, while using the X-ray classification it is $7.3 \times 10^{21} \mathrm{~cm}^{-2}$. Similarly, in the lowest luminosity bin, $L_{X}<3.2 \times 10^{42} \mathrm{erg} \mathrm{s}^{-1}$, obscured and unobscured AGNs are now clearly separated in $N_{\mathrm{H}}$. This separation is not even larger since heavily obscured low-luminosity sources are preferentially missed by the X-ray observations, and thus not included in our sample.

\subsection{Rest-Frame Optical Colors}

As is well known (e.g., Baldry et al. 2004; Weiner et al. 2005; Cirasuolo et al. 2007 and references therein), the distribution of optical colors in normal galaxies is bimodal, with a large population of blue star-forming galaxies in the blue cloud separated from the "red sequence" of massive passively evolving spheroids. As was recently reported (e.g., Barger et al. 2003; Nandra et al. 2007; Georgakakis et al. 2008; Silverman et al. 2008), most galaxies hosting obscured AGNs, in which the integrated optical light is dominated by the host galaxy (e.g., Treister et al. 2005), lie on the "green valley," a transition region located between the red sequence and the blue cloud. Hence, this can be considered as good evidence for a link between AGNs and galaxy evolution, possibly indicating that AGN feedback can play a role in regulating star formation (Springel et al. 2005; Schawinski et al. 2006); however, this remains controversial, as discussed by Alonso-Herrero et al. (2008).

To compute the rest-frame optical colors for the X-ray sources with spectroscopic identification on the ECDF-S field we started from the MUSYC optical catalog of Gawiser et al. (2006b). In order to increase the number of sources in our sample we added the X-ray sources with reliable photometric redshifts from the COMBO-17 survey, as described before. We then generated the rest-frame spectral energy distribution (SED) for each X-ray source with the measured redshift by interpolating the observed $U B V R I z J K_{s}$ photometric data points. The rest-frame $U$ and $V$ optical magnitudes were then computed by convolving the 

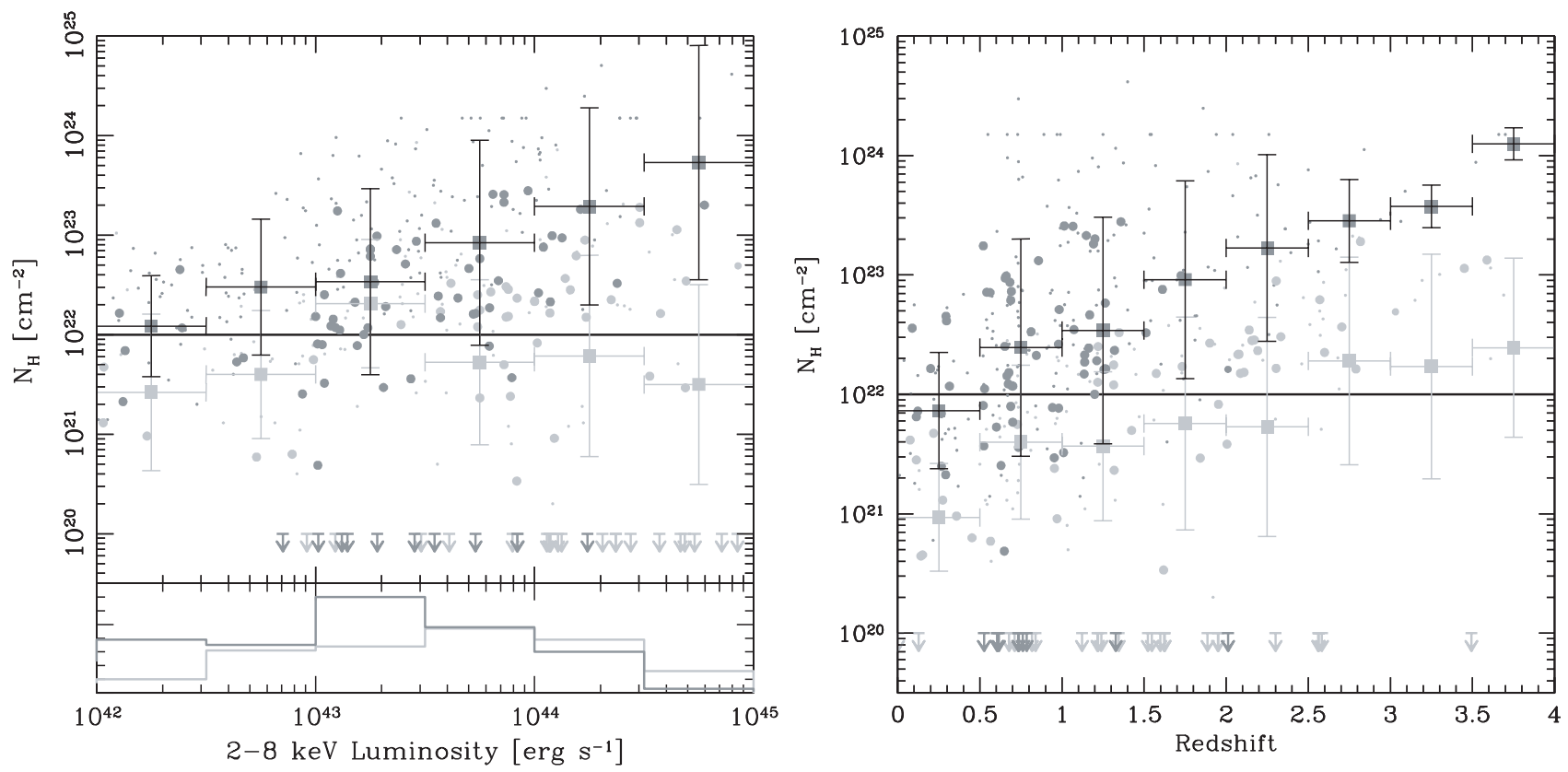

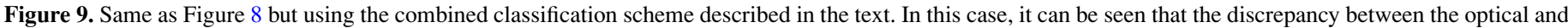
$\mathrm{X}$-ray classifications for low-luminosity sources is solved and our hybrid classification clearly separate sources in their average $N_{\mathrm{H}}$ values.

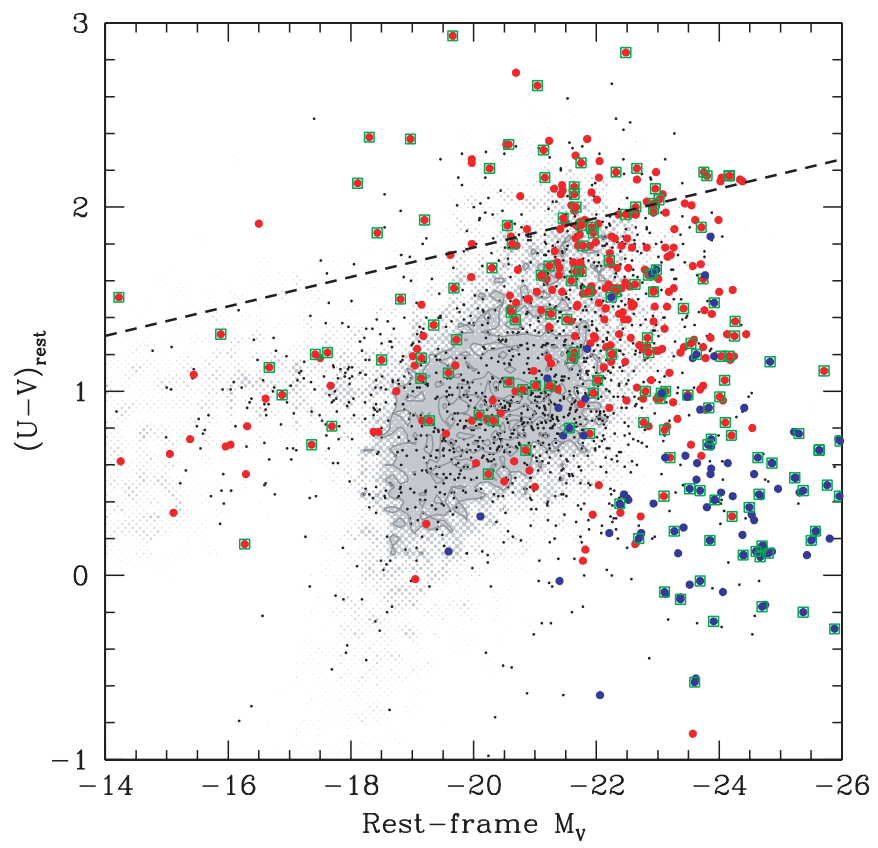

Figure 10. Rest frame $U-V$ color vs. rest-frame $V$-band absolute magnitude for obscured (red circles) and unobscured AGNs (blue circles). The green boxes mark the location of sources with spectroscopic identifications. Normal galaxies (i.e., non-AGN) with spectroscopic redshifts are shown by black circles, while the gray background shows the density of normal galaxies with photometric redshifts from COMBO-17. Contours enclose regions with densities larger than 10,20 , and 30 galaxies per interval. While the unobscured AGNs are in general bluer and have higher luminosities than normal galaxies, obscured AGNs have similar optical luminosities but have redder $U-V$ colors and live around the "green valley," shown by the dashed line, as determined by Bell et al. (2004) for $z=1$.

(A color version of this figure is available in the online journal.)

energy distribution with the corresponding filter response. The resulting rest-frame $U-V$ colors versus $M_{V}$, the rest-frame $V$ band absolute magnitude, are shown in Figure 10. A significant number of X-ray sources have $U-V<1$ and $M_{V}<-22$. In general, these sources were classified as unobscured AGNs because of the presence of broad emission lines. Since the optical continuum of these sources is dominated by the AGNs and not the host galaxy, we exclude them from further analysis. However, as it was found by Schawinski et al. (2009), once the emission from the central source is removed, the host galaxies of unobscured AGNs have optical colors similar to those of obscured AGNs. As can be seen by comparing with a population of normal galaxies obtained on the same field from the COMBO17 survey, the X-ray sources classified as obscured AGNs have in general redder optical colors than normal galaxies and lie outside the main blue cloud.

The excess of red sources in the AGN population can be easily seen in Figure 11, where the distributions of rest-frame $U-V$ colors for X-ray emitting sources and normal galaxies are presented. In order to limit the influence of differences in the sample selection, the comparison sample was obtained randomly from the ECDF-S inactive galaxies to match the AGN redshift distribution. Performing a KS test we found that the probability that these two distributions were drawn from the same parent distribution is negligible. Contrarily, a KS test comparing the distribution of the X-ray sources with spectroscopic and photometric redshift returned a relatively high probability of $\sim 20 \%$, indicating that the use of photometric redshifts does not significantly bias our results. The fact that results of the KS test are not even higher can be explained since in general sources with spectroscopic redshifts have brighter optical counterpart, and thus slightly different average $U-V$ colors. However, this is a minor effect that will not affect our conclusions.

Given that the comparison sample was selected to have the same absolute optical magnitude and redshift distributions as the AGN host galaxies, it is unlikely that the differences in optical colors are due to the sample selection. A similar analysis was performed by Georgakakis et al. (2008) using the AGNs detected in the AEGIS survey. Studying the morphology of AGN host galaxies, they found only a small fraction of major mergers, but evidence of minor interactions in a large number of sources. Both Georgakakis et al. (2008) and Schawinski et al. (2009) 


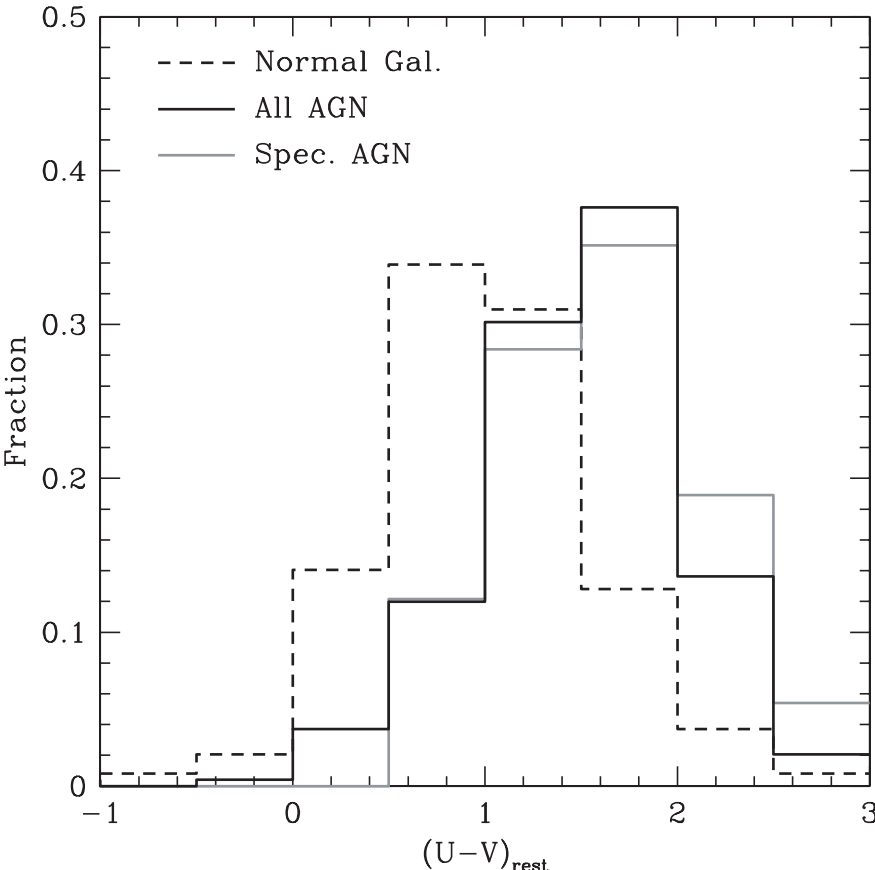

Figure 11. Normalized distribution of $U-V$ values for normal galaxies (dashed line), AGN (black solid line) and AGNs with spectroscopic identification (gray line) for sources with $-23<M_{V}<-18$. The sample of normal galaxies was selected randomly to match the AGN redshift distribution. The separation between normal galaxies and AGNs in their $U-V$ colors can be seen clearly. In this range of optical luminosities, most of the AGNs are obscured, hence the integrated emission is dominated by the galaxy, thus indicating that in general AGN host galaxies have redder color than nonactive galaxies.

concluded that AGN activity should outlive the truncation of star formation, in order to explain the observed red optical colors of AGN host galaxies.

\subsection{Obscured AGN Fraction}

The fraction of obscured AGNs and its possible dependence on parameters such as redshift or luminosity is very important for AGN population studies, in particular given its direct relation with our understanding of the cosmic XRB. A dependence of the obscured fraction on luminosity was previously found for X-rayselected samples by Ueda et al. (2003), Steffen et al. (2003), La Franca et al. (2005), and Treister et al. (2005) among others. A physical explanation, proposed by Lawrence (1991) and updated by Simpson (2005), is the so-called "receding torus." More recently, Treister et al. (2008) found a dependence of the ratio of mid-IR to bolometric flux on luminosity for unobscured AGNs consistent with an increase in the opening angle with luminosity. Similarly, Akylas \& Georgantopoulos (2008) proposed that the observed dependence of the obscured fraction on luminosity could be explained by the effects of photoionization on the Xray obscuring matter, while Hönig \& Beckert (2007) argues that the effects of the Eddington limit on a clumpy torus can explain the observed luminosity dependence of the obscured AGN fraction. The large sample of X-ray sources in the ECDF$\mathrm{S}$ can be used to further explore this luminosity dependence of the obscured AGN fraction. In Figure 12, we can see that using the ECDF-S sample alone the fraction of obscured AGN decreases from $\sim 90 \%$ at $L_{X}<10^{43} \mathrm{erg} \mathrm{s}^{-1}$ to $\sim 20 \%$ at $L_{X}=$ $10^{45} \mathrm{erg} \mathrm{s}^{-1}$, using the "hybrid" classification scheme described in Section 4.1.

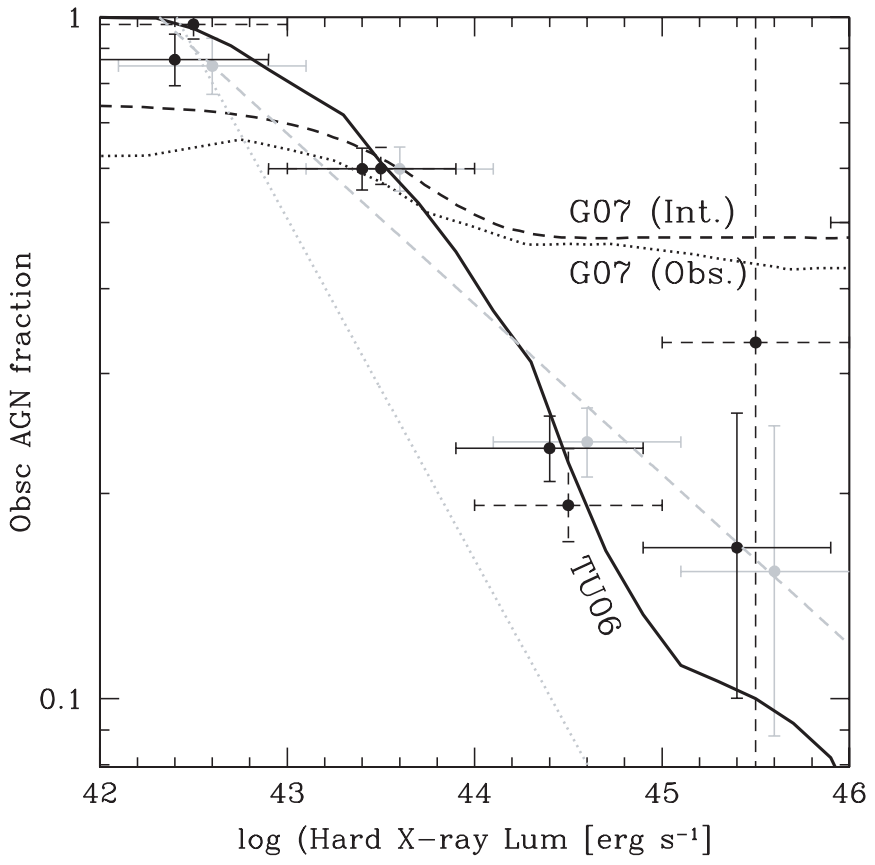

Figure 12. Ratio of obscured to total AGNs as a function of hard X-ray luminosity. The black circles with dashed error bars show the obscured AGN fraction from the ECDF-S alone, while the gray circles show the results obtained using the sample of Treister \& Urry (2006). Black circles with solid error bars show the fraction of obscured AGNs for the combined sample of Treister \& Urry (2006) and the ECDF-S sources. The dependence used by the Gilli et al. (2007) AGN population synthesis model for the intrinsic and observed fractions of obscured AGNs are shown by the dashed and dotted lines. The dependence used in the model presented by Treister \& Urry (2006) is shown by the solid line. While a good agreement is found between the observations and the expectations of Treister \& Urry (2006), the Gilli et al. (2007) model predicts a relatively large fraction of obscured AGNs, 50\%, at high luminosities, while the observed value is only $\sim 20 \%$. The dashed gray line shows the expected dependence for a radiation-limited torus as described by Hönig \& Beckert (2007), while the dotted gray line shows the expectation for the original receding torus of Lawrence (1991), both normalized to the observed value in the $10^{42}-10^{43} \mathrm{erg}$ $\mathrm{s}^{-1}$ bin.

In order to increase the significance of this result, we added the results from the ECDF-S to the compilation presented by Treister \& Urry (2006), which combined the results from seven $\mathrm{X}$-ray surveys ranging from wide area shallow surveys to the Chandra deep fields. The total sample includes now 2814 X-ray sources, 1377 (49\%) of them with spectroscopic identification. In Figure 12, we present the resulting obscured AGN fraction as a function of luminosity for the total sample. The obscured fraction ranges from $80 \pm 7 \%$ at $L_{X}<10^{43} \mathrm{erg} \mathrm{s}^{-1}$ to $16 \pm$ $8 \%$ for $L_{X}>10^{45} \mathrm{erg} \mathrm{s}^{-1}$. In addition, Figure 12 shows the predicted luminosity dependence incorporated in the Treister \& Urry (2005) AGN population synthesis model, as updated by Treister \& Urry (2006) to include a redshift dependence, and for comparison the luminosity dependence used in the models of Gilli et al. (2007). While at low luminosities both models agree well with the observations, for luminosities higher than $L_{X} \simeq$ $10^{44} \mathrm{erg} \mathrm{s}^{-1}$, the Gilli et al. (2007) models predict a fraction of obscured AGNs of $\sim 50 \%$, significantly higher than the observed value. This discrepancy has important consequences for the modeling of the XRB using AGN, as the largest contribution comes from sources at roughly these luminosities (e.g., Treister $\&$ Urry 2005). The larger fraction assumed by Gilli et al. (2007) is more relevant at lower energies, where the effects of absorption are larger. For example, the XRB intensity at $E<$ $10 \mathrm{keV}$ in the Gilli et al. (2007) model is $\sim 40 \%$ lower than the 
Treister \& Urry (2005) calculation, while it is now clear from recent Chandra and XMM observations (De Luca \& Molendi 2004; Hickox \& Markevitch 2006) that a larger XRB intensity at low energies is more appropriate.

In addition, in Figure 12 we compare the observed dependence of the fraction of obscured sources on luminosity with the expectations for different geometrical parameters of the obscuring material. If the height of the torus is roughly independent of luminosity, the change in covering fraction is due to a change in inner radius (the original "receding torus" model), hence a rough $L^{-1 / 2}$ dependence for the contrast should be expected (Barvainis 1987; Lawrence 1991). If the effects of radiation pressure are incorporated, in the case of a clumpy torus, Hönig \& Beckert (2007) derive a $L^{-1 / 4}$ dependence for the contrast. As can be seen in Figure 12, a $L^{-1 / 2}$ dependence is too steep compared to observed data. This implies that the height of the obscuring material cannot be independent of the source luminosity and provides evidence for a radiation-limited structure, as the one suggested by Hönig \& Beckert (2007).

The dependence of the fraction of obscured AGNs on redshift is more controversial. While some studies (e.g., La Franca et al. 2005; Ballantyne et al. 2006; Treister \& Urry 2006; Della Ceca et al. 2008) found a small increase in the fraction of obscured AGNs at higher redshifts, other results suggest that this fraction is constant (e.g., Ueda et al. 2003; Akylas et al. 2006). The upper panel of Figure 13 shows the observed fraction of obscured AGNs as a function of redshift for the sources in the ECDF-S. This fraction is high, $\sim 90 \%$, at $z<1$, while at higher redshifts it decreases to $\sim 30 \%-40 \%$. As for the luminosity dependence, in order to increase the significance of our results we added the ECDF-S sources to the sample of Treister \& Urry (2006). The results for the large sample are consistent with those from the ECDF-S alone, namely, at low redshift there is a large fraction of obscured sources with a steep decline at $z \sim 1$. This decline in the observed fraction of obscured sources can be easily explained by a simple observational fact: in order to be included in this sample a source needs to have a measured redshift from optical spectroscopy. For obscured AGNs, the optical light is dominated by the host galaxy (e.g., Barger et al. 2005; Treister et al. 2005), which becomes too faint for spectroscopy with $8 \mathrm{~m}$ class telescopes at $z \sim 1$. In order to quantify this effect, we use the ratio of identified to total X-ray sources in a given optical magnitude bin (upper panel, Figure 2). This magnitude-dependent ratio is used to calculate the expected obscured AGN fraction including the X-ray and optical flux limits and the luminosity dependence of the obscured fraction. This calculation is done as described in detail by Treister \& Urry (2006). Briefly, we used the AGN population synthesis of Treister \& Urry (2005), together with the library of AGN spectra described by Treister et al. (2004), and calculated the effective area of the survey as a function of both X-ray flux and optical magnitude, taking into account the spectroscopic incompleteness at each optical flux. This procedure outputs an expected number of observed obscured and unobscured AGNs as a function of redshift, for a nonevolving obscured AGN fraction.

As can be seen in the upper panel of Figure 13, the expected decrease is actually steeper than what is observed. This implies that the inferred fraction of obscured AGNs in the ECDF-S should increase with redshift, once the selection effects in the sample are accounted for. This dependence is consistent with the results of Treister \& Urry (2006), who found that this increase can be represented as a power law of the form $(1+z)^{\alpha}$, with $\alpha=$

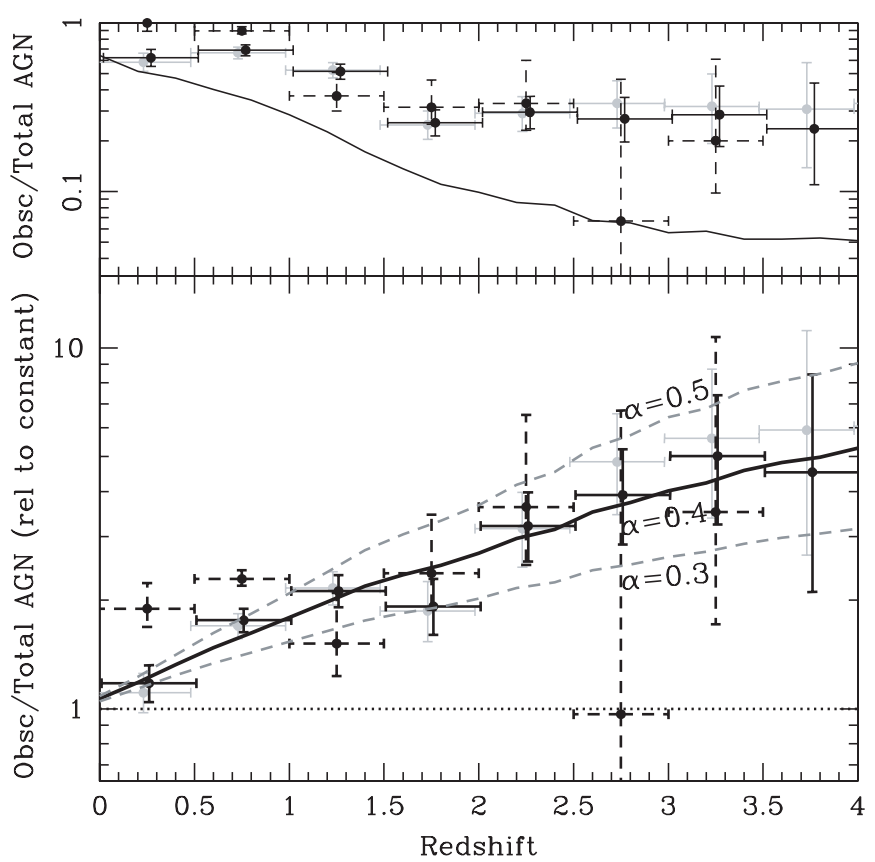

Figure 13. Fraction of obscured AGNs as a function of redshift. Upper panel: direct measurements using the sources on the ECDF-S field only (black circles with dashed error bars), from the Treister \& Urry (2006) sample (gray circles) and combining both samples (black circles with solid error bars). The expected observed fraction for an intrinsic fraction of 3:1 obscured to unobscured AGNs including optical and X-ray selection effects is shown by the black solid line. As can be seen, while the observed fraction of obscured AGNs declines toward higher redshifts, if the X-ray and optical selection effects and the luminosity dependence of the obscured AGN fraction are taken into account this decline should be even stronger. Bottom panel: inferred fraction of obscured AGNs relative to an intrinsically constant fraction after correcting for selection effect and including the luminosity dependence of the obscured AGN fraction. Symbols are the same as for the upper panel. The corrected fraction of obscured AGNs increases with redshift following a power law of the form $(1+z)^{\alpha}$ with $\alpha=0.4 \pm 0.1$, consistent with the results found by Treister \& Urry (2006).

$0.4 \pm 0.1$. These results are roughly independent of the method used to classify AGNs; we obtained the same dependence using both our mixed X-ray/optical classification scheme and one based completely on optical spectroscopy.

\subsection{Number Density and Evolution of AGNs}

Using our combined sample of X-ray-selected AGNs we can also compute the AGN spatial density as a function of redshift, and compare with the expectations from existing AGN luminosity functions (LFs) based on smaller samples. In order to calculate the AGN spatial density, we started from our collected sample of 1377 sources described above. We then separated the sources in low $\left(L_{X}=10^{41.5}-10^{43} \mathrm{erg} \mathrm{s}^{-1}\right)$, medium $\left(L_{X}=\right.$ $\left.10^{43}-10^{44.5} \mathrm{erg} \mathrm{s}^{-1}\right)$, and high luminosity $\left(L_{X}=10^{44.5}-10^{48} \mathrm{erg}\right.$ $\mathrm{s}^{-1}$ ) bins. For each luminosity class, we binned the sample in redshift so that each bin has at least 50 sources ( 20 for the highest luminosity sources). Then, the spatial density of each bin was calculated by summing the values of $V_{c}^{-1}$ for each source in the bin, where $V_{c}$ is the total comoving volume per unit area in that bin multiplied by the area covered by our supersample at the $\mathrm{X}$-ray flux of the source. In addition, upper limits for the spatial density on each bin were calculated by multiplying the values of $V_{c}$ by the fraction of spectroscopically identified sources at the optical magnitude of the AGN, as described in Treister \& Urry (2006) for the general sample and in the upper panel of Figure 2 for the ECDF-S. 


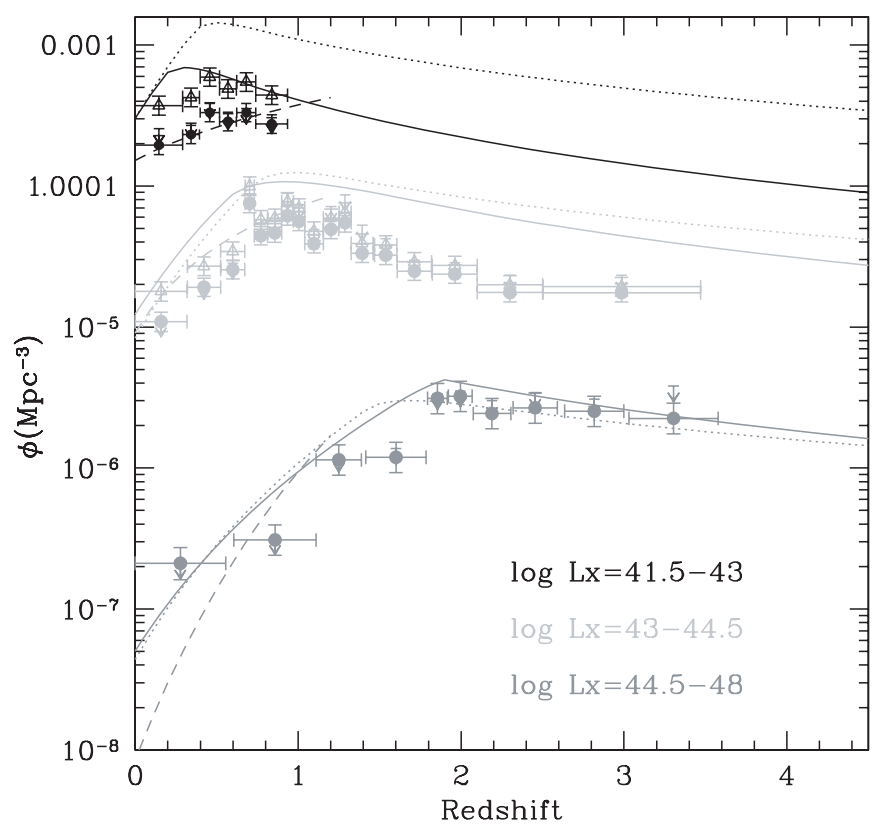

Figure 14. Space density of AGNs as a function of redshift derived from the sources in the ECDF-S. The filled circles show the observed density vs. $z$ in three luminosity bins, $\log L_{X}=41.5-43,43-44.5$, and 44.5-48 $\mathrm{erg} \mathrm{s}^{-1}$. The arrows show the effects of correcting these values for optical incompleteness effects, while the empty triangles show the results using the $N^{o b s} / N^{m d l}$ method to correct for incompleteness in the X-ray data. The lines show the AGN space density derived using the LFs of Ueda et al. (2003; solid lines), Barger et al. (2005; dashed lines), and La Franca et al. (2005; dotted lines). The Barger et al. (2005) LF is only defined for $z<1.2$. While in the highest luminosity bin all the studied LFs agree very well with the observations, at intermediate luminosities a good agreement is found only at $z<1$. In the lowest luminosity bin, the effects of incompleteness are more important, and hence only after correcting for them a good agreement with the LF of Ueda et al. (2003) is found. The Barger et al. (2005) LF was not corrected for incompleteness.

In Figure 14, we show the observed AGN spatial density as a function of redshift for low, medium, and high luminosity sources compared with the expected density obtained from integrating the hard X-ray LFs presented by Ueda et al. (2003), Barger et al. (2005), and La Franca et al. (2005). While the $\mathrm{X}$-ray sources in these studies were selected in similar ways, the samples have different number of sources (247 AGN in Ueda et al. 2003; 746 in Barger et al. 2005; and 508 in La Franca et al. 2005) with the emphasis set at different flux levels (mostly bright sources in Ueda et al. 2003; moderate fluxes in La Franca et al. 2005; and faint sources in Barger et al. 2005). In addition, the modeling of the LF is also different in these papers. While Barger et al. (2005) assumed pure luminosity evolution, both Ueda et al. (2003) and La Franca et al. (2005) found better results using a luminosity-dependent density evolution. As can be seen in Figure 14, at high and moderate luminosities all the LFs studied here agree well with the observations at low redshifts, while at high redshifts the expectations from both the Ueda et al. (2003) and La Franca et al. (2005) works are significantly above the observed values for intermediate luminosity sources.

This discrepancy can most likely be explained by the effects of incompleteness in the X-ray data, which are particularly important for the lower luminosity sources. As it was concluded by, e.g., La Franca \& Cristiani (1997), the $V_{c}^{-1}$ method used here is particularly sensitive to the effects of incompleteness. The work of Ueda et al. (2003) and La Franca et al. (2005) both used the $N^{o b s} / N^{m d l}$ method, which attempts to account for incompleteness by using the expected number of sources in a given luminosity and redshift bin. One obvious caveat of this method is that the results are model dependent. We attempt to correct for incompleteness in the X-ray data, in the two lowest luminosity bins, where these effects are more important. In order to do that, we use the model described in the previous section in order to calculate the fraction of sources missed by the X-ray selection in a given luminosity and redshift bin due to the effects of obscuration. For the lowest luminosity sources, this correction is roughly $\sim 2$ times, while at higher luminosities it is about $30 \%-40 \%$. We did not attempt to correct the highest luminosity bin, since the effects of absorption are negligible for these sources. As can be seen in Figure 14, after the correction for incompleteness is applied a good agreement with the predicted spatial density using the LF of Ueda et al. (2003) is found. The remaining discrepancy with the work of La Franca et al. (2005) could be explained because in this case even highly obscured Compton-thick AGNs up to $N_{\mathrm{H}}=10^{26}$ $\mathrm{cm}^{-2}$ are included, while in the case of Ueda et al. (2003) and in our work only mildly Compton-thick AGNs with $N_{\mathrm{H}}<10^{25}$ $\mathrm{cm}^{-2}$ are considered. Finally, it is worth mentioning that in the LF of Barger et al. (2005) no attempts were made to correct for incompleteness in the X-ray selection. Hence, a good agreement is found only for the uncorrected data points.

\subsection{Infrared (IR) to X-Ray Ratio}

Emission at X-ray wavelengths, in particular in the hard band, is often used as a tracer for the direct AGN output mainly from Compton-scattered accretion disk photons, while the luminosity at longer wavelengths, in particular in the mid-far infrared, is associated with the reradiation of the energy absorbed by the surrounding gas and dust (e.g., Pier \& Krolik 1993). There is currently a strong debate about the geometry and characteristics of this surrounding dust, in particular whether it has a smooth (e.g., Pier \& Krolik 1992) or clumpy (e.g., Krolik \& Begelman 1988) distribution. The ratio of IR to X-ray luminosity can be used to distinguish between these two distributions and to constrain the dust geometry (Lutz et al. 2004). For example, smooth torus models predict large differences in the IR to X-ray ratio for obscured and unobscured sources, due to the significant effects of self-absorption. Contrarily, radiation transfer models of clumpy dust torii predict very small or no differences between the IR to X-ray ratio for obscured and unobscured AGNs.

The observational evidence remains controversial. The work of Horst et al. (2006) reports that no differences were found in the mid-IR (measured at a fiducial rest-frame wavelength of $12.3 \mu \mathrm{m}$ ) to X-ray ratio for a sample of 17 nearby AGNs observed with the VLT-VISIR, which provides a relatively high angular resolution of $\sim 0^{\prime \prime} 35$. In apparent contradiction, Ramos Almeida et al. (2007) found a slightly smaller value for the $\mathrm{X}$-ray to nuclear mid-IR (at $6.75 \mu \mathrm{m}$ ) ratio from a sample of 57 AGNs. For the latter, the observations were carried out using the ISOCAM camera on board Infrared Space Observatory $(I S O)$, with a more limited angular resolution of $\sim 4^{\prime \prime}$. Horst et al. (2008) argue that precisely this difference in angular resolution explains the discrepant results. Using their high spatial resolution images, they claim that the contribution from star formation, unresolved in the ISOCAM observations, can account for the observed differences between obscured and unobscured AGNs. On the other hand, the relatively small sample of Horst et al. (2006) traces larger intrinsic luminosities for unobscured sources compared to the obscured sample, thus contributing to explain why no difference in the IR to X-ray ratio was found. Given the large scatter in the IR to X-ray ratio 
reported by both groups, a large sample of sources is required to reach statistically significant conclusions.

In Figure 15, we present the values of the ratio of $L_{\lambda}$ to $L_{X}$ as a function of $\lambda$, the rest-frame wavelength. These values were computed using both the IRAC and MIPS fluxes, for the sources in our sample with Spitzer detections and $N_{\mathrm{H}}$ measurements. We also added the sources in the central region with $N_{\mathrm{H}}$ measured from the X-ray spectrum by Tozzi et al. (2006). In order to correct the hard X-ray $(2-8 \mathrm{keV})$ luminosities, $L_{X}$, for the effects of absorption we used the photoelectric absorption cross sections derived by Morrison \& McCammon (1983) and the $N_{\mathrm{H}}$ values measured from $\mathrm{X}$-ray spectral fitting as described above. Given that the effects of X-ray absorption in sources classified as unobscured are typically very small, and those values can be significantly affected by measurement errors, we only corrected the X-ray luminosities of the obscured sources. If no correction for absorption is done to the $\mathrm{X}$-ray luminosities of the obscured sources, our conclusions are unchanged. As can be seen in Figure 15, sources classified as obscured and unobscured AGNs have significantly different average values of $L_{\lambda} / L_{X}$, and this separation depends on wavelength. At the shortest wavelengths, $\lambda \sim 1 \mu \mathrm{m}$, the separation in this ratio is rather small, which can be explained by the contribution of the stellar light of the host galaxy to the integrated emission. This effect remains visible until $\lambda \sim 2 \mu \mathrm{m}$, where the stellar light starts to fade and emission from the host dust in the inner region of the surrounding material in the AGNs begins to dominate. This emission is highly affected by self-absorption, as most torus models predict (see e.g., Pier \& Krolik 1993; Nenkova et al. 2002; Hönig et al. 2006), thus explaining why at these wavelengths unobscured sources have significantly higher values of $L_{\lambda} / L_{X}$. At longer wavelengths, $\lambda \gtrsim$ $10 \mu \mathrm{m}$, the contrast between obscured and unobscured sources is reduced again, since the optical depth is reduced at longer wavelengths. Thus, it is expected that at $\lambda \sim 30-40 \mu \mathrm{m}$ the IR emission should become isotropic again.

In order to compare with the observed averages for nearby Seyfert galaxies, in Figure 15 we also present the composite IR spectrum for local sources classified as Seyfert 1 (unobscured) and Seyfert 2 (obscured), as compiled by Polletta et al. (2007), normalized at a fiducial wavelength of $100 \mu \mathrm{m}$, where the IR re-emission is expected to be fully isotropic. It is remarkable that both composite spectra agree well with our average values, thus indicating that the sources in our sample, most of them at $z=0.5-1$ have very similar IR spectra to local active galaxies and higher luminosity sources. In addition, the results presented in Figure 15 allow to explain why Ramos Almeida et al. (2007) and Horst et al. (2008) reach apparently discrepant conclusions. While the work of Ramos Almeida et al. (2007) was based on observations at $\sim 6 \mu \mathrm{m}$, where the contrast between obscured and unobscured sources is nearly maximal, Horst et al. (2008) used observations of a limited sample of sources at $\sim 12 \mu \mathrm{m}$, where the contrast is smaller than at shorter wavelengths. Thus, it is possible that the results of Horst et al. (2008) are dominated by the intrinsic scatter in this relation, in particular given the low number of sources in their sample.

In Figure 15, we also compare the observed infrared luminosities with the predictions from torus models recently presented by Nenkova et al. (2008). These models assume a clumpy distribution for the obscuring material. While establishing the physical parameters of the dust surrounding the central region is a difficult task, which is beyond the scope of this paper, in Figure 15 we show examples of the expected IR spectrum for obscured and unobscured sources. The assumed parameters

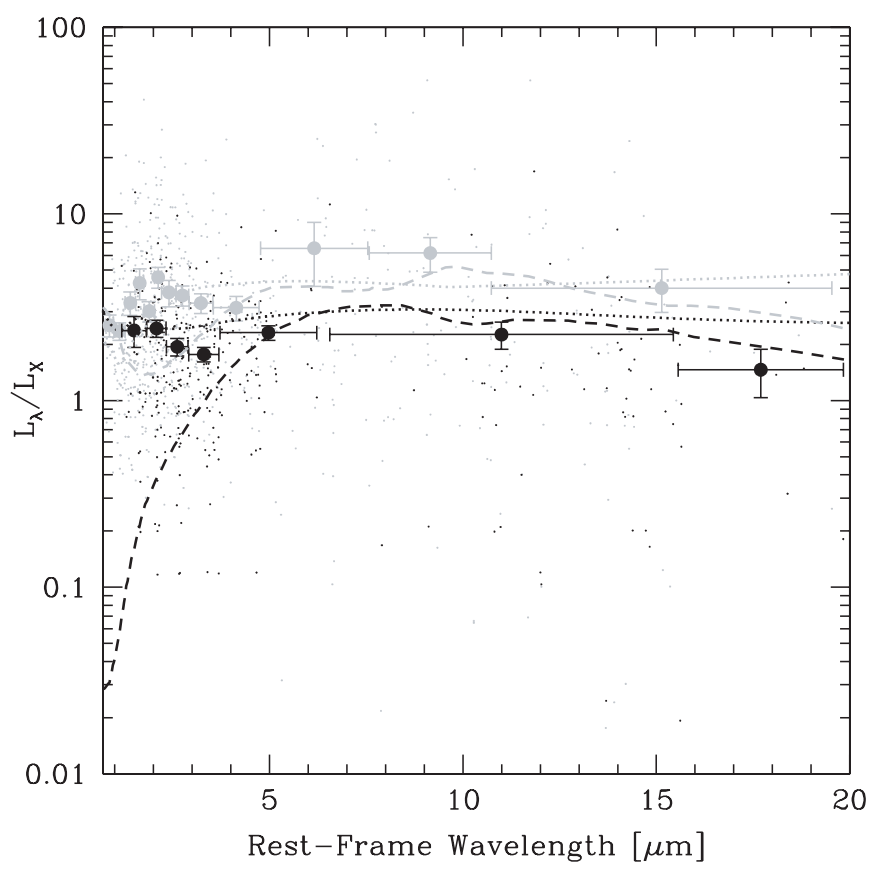

Figure 15. Monochromatic luminosity normalized by the hard X-ray luminosity as a function of rest-frame wavelength for obscured (small black circles) and unobscured (small light gray circles) AGNs. For each source the four Spitzer IRAC and the MIPS-24 $\mu \mathrm{m}$ photometric data points were used. The large circles with error bars show the average values for each AGN type, in bins that include 40 sources. The dotted lines show the composite spectra for obscured and unobscured quasars, as compiled by Polletta et al. (2007), while the dashed lines show examples of expected IR spectrum from the clumpy torus models of Nenkova et al. (2008), as described in the text. A clear separation between the average values for obscured and unobscured sources is observed at wavelengths $\sim 5-15 \mu \mathrm{m}$, which can be explained by the effects of self-absorption by the surrounding material in the obscured sources.

are described by Nenkova et al. (2008) in the caption of their Figure 4, for a $r^{-3}$ radial distribution of clouds. Following the basic assumption of the original AGN unification paradigm, the only difference between the obscured and unobscured model is the viewing angle. As can be seen, a decent agreement is found between the model spectrum and observations, in particular at wavelengths longer than $\sim 5 \mu \mathrm{m}$. At shorter wavelengths, the additional contribution from the AGN host galaxy (not included in the model spectrum) starts to become significant, in particular for the obscured sources.

Taking advantage of the large number of sources in our sample, in Figure 16 we show $L_{\lambda} / L_{X}$ as a function of wavelength for sources separated in three luminosity bins: $L_{X}<10^{43} \mathrm{erg}$ $\mathrm{s}^{-1}, 10^{43} \mathrm{erg} \mathrm{s}^{-1}<L_{X}<10^{44} \mathrm{erg} \mathrm{s}^{-1}$, and $L_{X}>10^{44} \mathrm{erg} \mathrm{s}^{-1}$. A clear conclusion from this figure is that the difference in the average ratio between obscured and unobscured sources is largest at the lowest luminosity bin, while for higher luminosity sources the $L_{\lambda} / L_{X}$ ratios become very similar. In their study of the mid-IR properties of nearby AGNs, Polletta et al. (2007) found somewhat consistent results. According to their Figure 9, the ratio $L_{I R}$ (at rest-frame $6 \mu \mathrm{m}$ ) to $L_{X}$ for obscured sources, obtained combining their AGN2 and star-forming (SF) classes, is significantly lower than the ratio for unobscured AGNs (their AGN1 class). In our interpretation of Figure 16, this increasing contrast for lower luminosity sources can be understood in terms of the luminosity dependence of the geometrical parameters of the absorbing region, in particular the opening angle, as described by Treister et al. (2008). 


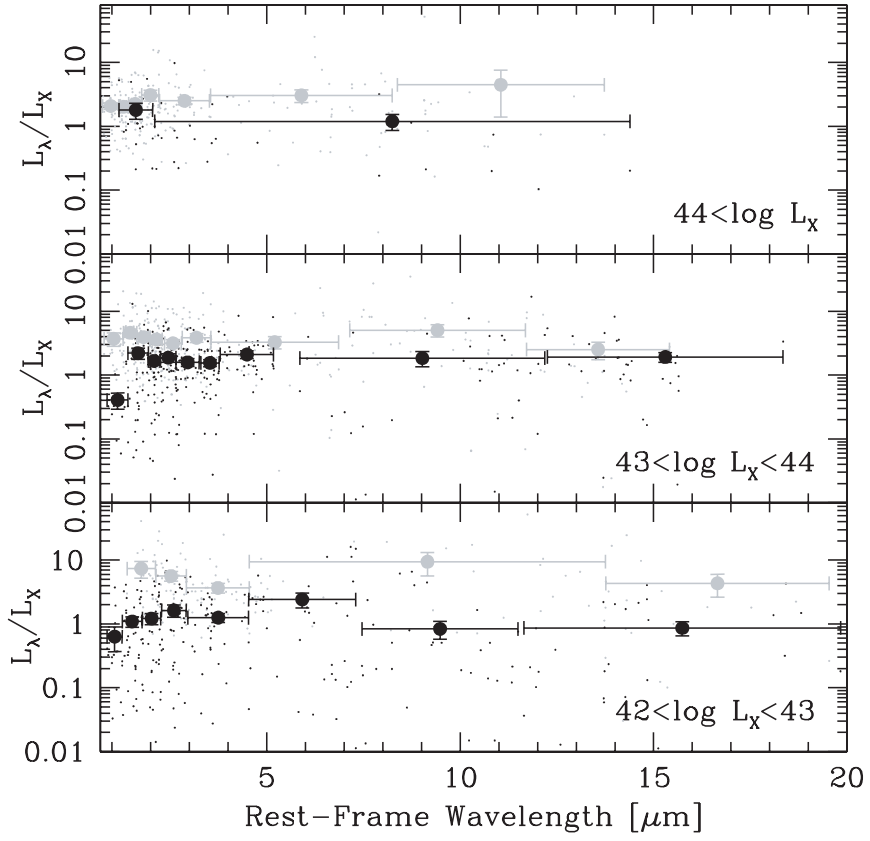

Figure 16. Same as Figure 15 but separating the sample in three luminosity bins: $\log L_{X}=42-43,43-44$, and $>44 \mathrm{erg} \mathrm{s}^{-1}$. The largest separation between obscured and unobscured sources is found at the lowest luminosities, while for more luminous sources this difference is smaller and almost negligible. One way to interpret this result is including a dependence of the opening angle on luminosity, as found by Treister et al. (2008), such that the lower luminosity sources have larger amounts of self-absorption due to a smaller opening angle.

\section{CONCLUSIONS}

We presented here the first results from our optical spectroscopy program, aimed to provide identifications and redshifts for the X-ray sources detected by Chandra in the ECDF-S field. As part of this work we targeted $339 \mathrm{X}$-ray sources from the catalog of Virani et al. (2006) and obtained redshifts and identifications for 160 sources. We also added 26 sources with identifications obtained from the literature. While most of the sources with broad emission lines are found at $z>1$, the mean redshift for the sources in our sample is 0.75 . In order to separate obscured and unobscured AGNs we adopted a mixed scheme, combining the optical spectroscopy information with the indication of the X-ray spectrum given by the HR. This hybrid scheme agrees very well in most cases with the amount of neutral hydrogen column density measured in the X-ray spectrum for the brightest sources.

The optical colors of the obscured and/or low-luminosity AGNs are dominated by the host galaxy, and are on average redder than nonactive galaxies, as was found previously by other authors. We also focused on the fraction of obscured AGNs and its possible dependence on luminosity and redshift. For the latter, we confirmed at higher significance the results of Treister \& Urry (2006), indicating that after correcting for selection effects the fraction of obscured AGNs increase with redshift. Regarding a possible luminosity dependence, we confirmed the functional form derived by Treister \& Urry (2005) and found that the population synthesis model of Gilli et al. (2007) significantly overestimates the fraction of obscured AGNs at high luminosities. We also computed the AGN spatial density as a function of redshift and found results in agreement with the expected values using the LF of Barger et al. (2005), but significant discrepancies with the LFs of Ueda et al. (2003) and La Franca et al. (2005), which can however be most likely explained by the effects of incompleteness in our X-ray-selected sample.

Taking advantage of the deep multiwavelength data available in the ECDF-S we studied the infrared properties of the X-rayselected AGNs with spectroscopic identifications. We found a significant difference in the fraction of bolometric light emitted at mid-IR wavelengths by obscured and unobscured AGNs. These differences can be explained by the effects of dust selfabsorption, for which the maximum contrast should be at $\sim 5-$ $15 \mu \mathrm{m}$. Furthermore, by separating our sample into luminosity bins we found that the contrast is larger for the lower luminosity sources. One possible interpretation for this result is that the opening angle is larger for high-luminosity sources, such that the effects of self-absorption are less important.

We are grateful for the help and support of the staff at Las Campanas and Paranal observatories, and for very useful discussions with Mark Gieles and Fabio La Franca. We thank Tom Aldcroft for adapting Yaxx to our specific needs. Support for the work of ET was provided by the National Aeronautics and Space Administration through Chandra Postdoctoral Fellowship Award Number PF8-90055 issued by the Chandra $\mathrm{X}$-ray Observatory Center, which is operated by the Smithsonian Astrophysical Observatory for and on behalf of the National Aeronautics Space Administration under contract NAS8-03060. S.V., C.M.U., and C.N.C. acknowledge support from NSF grant No. AST0407295 and Spitzer JPL grant No. RSA1288440. S.V. acknowledges support from a graduate research scholarship awarded by the Natural Science and Engineering Research Council of Canada (NSERC), a Grant-in-Aid of Research from the National Academy of Sciences (administered by Sigma Xi, The Scientific Research Society), and from NASA/INTEGRAL grant NNG05GM79G. This work is based on observations made with the $6.5 \mathrm{~m}$ Magellan telescopes, a collaboration between the Observatories of the Carnegie Institution of Washington, University of Arizona, Harvard University, University of Michigan, and Massachusetts Institute of Technology, and at Cerro Tololo Inter-American Observatory, a division of the National Optical Astronomy Observatories, which is operated by the Association of Universities for Research in Astronomy, Inc. under cooperative agreement with the National Science Foundation. This research has made use of NASA's Astrophysics Data System.

Facilities: CXO (ACIS), Magellan: Baade (IMACS), VLT: Melipal (VIMOS), Spitzer (IRAC, MIPS).

\section{REFERENCES}

Adami, C., et al. 2005, A\&A, 443, 805

Akylas, A., \& Georgantopoulos, I. 2008, A\&A, 479, 735

Akylas, A., Georgantopoulos, I., Georgakakis, A., Kitsionas, S., \& Hatziminaoglou, E. 2006, A\&A, 459, 693

Alonso-Herrero, A., Pérez-González, P. G., Rieke, G. H., Alexander, D. M., Rigby, J. R., Papovich, C., Donley, J. L., \& Rigopoulou, D. 2008, ApJ, 677, 127

Arnaud, K. A. 1996, in ASP Conf. Ser. 101, Astronomical Data Analysis Software and Systems V, ed. G. H. Jacoby \& J. Barnes (San Francisco, CA: ASP), 17

Baldry, I. K., Glazebrook, K., Brinkmann, J., Ivezić, Ž., Lupton, R. H., Nichol, R. C., \& Szalay, A. S. 2004, ApJ, 600, 681

Ballantyne, D. R., Everett, J. E., \& Murray, N. 2006, ApJ, 639, 740

Barger, A. J., Cowie, L. L., Mushotzky, R. F., Yang, Y., Wang, W.-H., Steffen, A. T., \& Capak, P. 2005, AJ, 129, 578

Barger, A. J., et al. 2003, AJ, 126, 632

Barvainis, R. 1987, ApJ, 320, 537

Bauer, F. E., Alexander, D. M., Brandt, W. N., Schneider, D. P., Treister, E., Hornschemeier, A. E., \& Garmire, G. P. 2004, AJ, 128, 2048 
Bell, E. F., et al. 2004, ApJ, 608, 752

Brandt, W. N., et al. 2001, AJ, 122, 2810

Cardamone, C. N., Moran, E. C., \& Kay, L. E. 2007, AJ, 134, 1263

Cardamone, C. N., et al. 2008, ApJ, 680, 130

Cirasuolo, M., et al. 2007, MNRAS, 380, 585

Comastri, A., Setti, G., Zamorani, G., \& Hasinger, G. 1995, A\&A, 296, 1

Croom, S. M., Warren, S. J., \& Glazebrook, K. 2001, MNRAS, 328, 150

Davis, M., et al. 2007, ApJ, 660, L1

De Luca, A., \& Molendi, S. 2004, A\&A, 419, 837

Della Ceca, R., et al. 2008, A\&A, 487, 119

Fazio, G. G., et al. 2004, ApJS, 154, 10

Gawiser, E., et al. 2006a, ApJS, 162, 1

Gawiser, E., et al. 2006b, ApJ, 642, L13

Georgakakis, A., et al. 2008, MNRAS, 385, 2049

Giacconi, R., et al. 2001, ApJ, 551, 624

Gilli, R., Comastri, A., \& Hasinger, G. 2007, A\&A, 463, 79

Gilli, R., Salvati, M., \& Hasinger, G. 2001, A\&A, 366, 407

Gilli, R., et al. 2003, ApJ, 592, 721

Gilli, R., et al. 2005, A\&A, 430, 811

Gruber, D. E. 1992, in The X-Ray Background, ed. X. Barcons \& A. C. Fabian (Cambridge: Cambridge Univ. Press), 44

Hasinger, G., Miyaji, T., \& Schmidt, M. 2005, A\&A, 441, 417

Hickox, R. C., \& Markevitch, M. 2006, ApJ, 645, 95

Hildebrandt, H., et al. 2006, A\&A, 452, 1121

Hönig, S. F., \& Beckert, T. 2007, MNRAS, 380, 1172

Hönig, S. F., Beckert, T., Ohnaka, K., \& Weigelt, G. 2006, A\&A, 452, 459

Horst, H., Gandhi, P., Smette, A., \& Duschl, W. J. 2008, A\&A, 479, 389

Horst, H., Smette, A., Gandhi, P., \& Duschl, W. J. 2006, A\&A, 457, L17

Kalberla, P. M. W., Burton, W. B., Hartmann, D., Arnal, E. M., Bajaja, E., Morras, R., \& Pöppel, W. G. L. 2005, A\&A, 440, 775

Koekemoer, A. M., et al. 2007, ApJS, 172, 196

Krolik, J. H., \& Begelman, M. C. 1988, ApJ, 329, 702

La Franca, F., \& Cristiani, S. 1997, AJ, 113, 1517

La Franca, F., et al. 2005, ApJ, 635, 864

Lawrence, A. 1991, MNRAS, 252, 586

Le Fèvre, O., et al. 2003, in SPIE Conf. Ser. 4841, Instrument Design and Performance for Optical/Infrared Ground-based Telescopes, ed. M. Iye \& A. F. M. Moorwood (Bellingham, WA: SPIE), 1670

Le Fèvre, O., et al. 2004, A\&A, 428, 1043

Lehmer, B. D., et al. 2005, ApJS, 161, 21

Lira, P., Ward, M., Zezas, A., Alonso-Herrero, A., \& Ueno, S. 2002, MNRAS, 330,259

Lutz, D., Maiolino, R., Spoon, H. W. W., \& Moorwood, A. F. M. 2004, A\&A, 418,465

Makovoz, D., \& Marleau, F. R. 2005, PASP, 117, 1113

Mignano, A., et al. 2007, A\&A, 462, 553

Moran, E. C., Filippenko, A. V., \& Chornock, R. 2002, ApJ, 579, L71

Morrison, R., \& McCammon, D. 1983, ApJ, 270, 119
Nandra, K., George, I. M., Mushotzky, R. F., Turner, T. J., \& Yaqoob, T. 1997, ApJ, 476, 70

Nandra, K., \& Pounds, K. A. 1994, MNRAS, 268, 405

Nandra, K., et al. 2007, ApJ, 660, L11

Nenkova, M., Ivezić, Ž., \& Elitzur, M. 2002, ApJ, 570, L9

Nenkova, M., Sirocky, M. M., Nikutta, R., Ivezić, Ž., \& Elitzur, M. 2008, ApJ, 685,160

Oke, J. B., \& Gunn, J. E. 1983, ApJ, 266, 713

Pier, E. A., \& Krolik, J. H. 1992, ApJ, 401, 99

Pier, E. A., \& Krolik, J. H. 1993, ApJ, 418, 673

Polletta, M., et al. 2007, ApJ, 663, 81

Ramos Almeida, C., Pérez García, A. M., Acosta-Pulido, J. A., \& Rodríguez Espinosa, J. M. 2007, AJ, 134, 2006

Rieke, G. H., et al. 2004, ApJS, 154, 25

Risaliti, G., Maiolino, R., \& Salvati, M. 1999, ApJ, 522, 157

Rosati, P., et al. 2002, ApJ, 566, 667

Schawinski, K., Virani, S., Simmons, B., Urry, C.M., Treister, E., \& Kaviraj, S. 2009, ApJ, 692, L19

Schawinski, K., et al. 2006, Nature, 442, 888

Schneider, D. P., et al. 2002, AJ, 123, 567

Scoville, N., et al. 2007, ApJS, 172, 1

Setti, G., \& Woltjer, L. 1989, A\&A, 224, L21

Silverman, J. D., et al. 2008, ApJ, 675, 1025

Simpson, C. 2005, MNRAS, 360, 565

Spergel, D. N., et al. 2007, ApJS, 170, 377

Springel, V., Di Matteo, T., \& Hernquist, L. 2005, MNRAS, 361, 776

Steffen, A. T., Barger, A. J., Cowie, L. L., Mushotzky, R. F., \& Yang, Y. 2003, ApJ, 596, L23

Szokoly, G. P., et al. 2004, ApJS, 155, 271

Taylor, E., et al. 2009, ApJS, submitted

Tozzi, P., et al. 2006, A\&A, 451, 457

Treister, E., Gawiser, E., van Dokkum, P., Lira, P., Urry, M., \& The Musyc Collaboration. 2007, The Messenger, 129, 45

Treister, E., Krolik, J. H., \& Dullemond, C. 2008, ApJ, 679, 140

Treister, E., \& Urry, C. M. 2005, ApJ, 630, 115

Treister, E., \& Urry, C. M. 2006, ApJ, 652, L79

Treister, E., et al. 2004, ApJ, 616, 123

Treister, E., et al. 2005, ApJ, 621, 104

Treister, E., et al. 2006, ApJ, 640, 603

Ueda, Y., Akiyama, M., Ohta, K., \& Miyaji, T. 2003, ApJ, 598, 886

Vanzella, E., et al. 2005, A\&A, 434, 53

Vanzella, E., et al. 2006, A\&A, 454, 423

Vanzella, E., et al. 2008, A\&A, 478, 83

Virani, S. N., Treister, E., Urry, C. M., \& Gawiser, E. 2006, AJ, 131, 2373

Weiner, B. J., et al. 2005, ApJ, 620, 595

Wolf, C., et al. 2004, A\&A, 421, 913

Zheng, W., et al. 2004, ApJS, 155, 73 